\title{
Statistical ensembles with finite bath: A description for an event generator
}

\author{
M. Hauer \\ Helmholtz Research School, University of Frankfurt, Frankfurt, Germany and UCT-CERN Research Centre and \\ Department of Physics, University of Cape Town, Rondebosch 7701, South Africa \\ S. Wheaton \\ UCT-CERN Research Centre and Department of Physics, University of Cape Town, Rondebosch 7701, South Africa
}

(Received 17 September 2009; published 30 November 2009)

\begin{abstract}
A Monte Carlo event generator has been developed assuming thermal production of hadrons. The system under consideration is sampled grand canonically in the Boltzmann approximation. A reweighting scheme is then introduced to account for conservation of charges (baryon number, strangeness, electric charge) and energy and momentum, effectively allowing for extrapolation of grand canonical results to the microcanonical limit. This method has two strong advantages compared to analytical approaches and standard microcanonical Monte Carlo techniques in that it is capable of handling resonance decays as well as (very) large system sizes.
\end{abstract}

DOI: $10.1103 /$ PhysRevC.80.054915

PACS number(s): 24.10.Pa, 24.60.Ky, 05.30.-d

\section{INTRODUCTION}

The statistical hadronization model, first introduced by Fermi [1] and Hagedorn [2], has been remarkably successful in the description of experimentally measured average hadron production yields in heavy-ion collisions ranging from the GSI Schwerionen Synchrotron (SIS) [3] and BNL Alternating Gradient Synchrotron (AGS) [4] over CERN Super Proton Synchrotron (SPS) [5] to BNL Relativistic Heavy Ion Collider (RHIC) [6] energies. Over time this has led to the establishment of the "chemical freeze-out line" [7], which is now a vital part of our understanding of the phase diagram of strongly interacting matter. Model predictions for the upcoming LHC and future FAIR [8,9] experiments largely follow these trends.

Somewhere above this freeze-out line in the phase diagram we expect, in general, a phase transition from hadronic degrees of freedom to a phase of deconfined quarks and gluons, generally termed the quark gluon plasma; and more specifically, a first-order phase transition at low temperature and high baryon chemical potential, and a crossover at high temperature and low baryon chemical potential. In between, a second-order endpoint or a critical point might emerge. For recent reviews, see Refs. [10,11].

Fluctuation and correlation observables are among the most promising candidates suggested to be suitable for signaling the formation of new states of matter and transitions between them. For recent reviews here see Refs. [12-15]. The statistical properties of a sample of events are, however, certainly not solely determined by critical phenomena. More broadly speaking, they depend strongly on the way events are chosen for the analysis, and on the information available about the system.

The ideal gas approximation of the statistical hadronization model will again serve as our testbed. Its strong advantage is that it is simple and, to some extent, intuitive. Given its success in describing experimentally measured average hadron yields, and its ability to reproduce low temperature lattice susceptibilities [16], the question arises as to whether fluctuation and correlation observables also follow its main line. Critical phenomena (and many more), however, remain beyond the present study.
Conventionally in statistical mechanics three standard ensembles are discussed; the microcanonical ensemble (MCE), the canonical ensemble (CE), and the grand canonical ensemble (GCE). In the $\mathrm{MCE}^{1}$ one considers an ensemble of microstates with exactly fixed values of extensive conserved quantities (energy, momentum, electric charge, etc.), with "a priori equal probabilities" of all microstates (see, e.g., Ref. [17]). The CE introduces the concept of temperature by introduction of an infinite thermal bath, which can exchange energy (and momentum) with the system. The GCE introduces further chemical potentials by attaching the system under consideration to an infinite charge bath. ${ }^{2}$ Only if the experimentally accessible system is just a small fraction of the total, and all parts have had the opportunity to mutually equilibrate, can the appropriate ensemble be the grand canonical ensemble.

A statistical hadronization model Monte Carlo event generator affords us with the possibility of studying fluctuation and correlation observables in equilibrium systems. Data analysis can be done in close relation to experimental analysis techniques. Imposing global constraints on a sample is always technically a bit more challenging. Direct sampling of MCE events (or microstates) has only been done in the nonrelativistic limit [18]. Sample and reject procedures, suitable for relativistic systems, become rapidly inefficient with large system size. However, they have the advantage of being very successful for small system sizes $[19,20]$.

In this article we try a different approach: we sample the GCE, then reweight events according to their values of extensive quantities, and approach the sample-reject limit (MCE) in a controlled manner. In this way one can study the statistical properties of a global equilibrium system in their dependence on the size of their thermodynamic bath. As any of the three standard ensembles remain idealizations

\footnotetext{
${ }^{1}$ The term MCE is also often applied to ensembles with energy but not momentum conservation.

${ }^{2}$ Note that a system with many charges can have some charges described via the CE and others via the GCE.
} 
of physical systems, one might find intermediate ensembles to be of phenomenological interest too.

We study the first and, in particular, second moments of joint distributions of extensive quantities. We concentrate mainly on particle number distributions and distributions of "conserved" charges and discuss the influence of acceptance cuts in momentum space, conservations laws, and resonance decay on the statistical properties of a sample of hadron resonance gas model events. We extend our previous studies of ideal particle and antiparticle gases [21,22] and of gases of altogether massless particles [23].

The numerical code has been written for inclusion into the already existing THERMUS package [24]. We make frequent use of the functionality provided by the ROOT framework [25].

The article is organized as follows: In Sec. II the basic ideas of this article are formulated. The GCE Monte Carlo sampling procedure is described in Sec. III. The first and second moments of the distributions of fully phase space integrated extensive quantities are then extrapolated to the microcanonical limit in Sec. IV. Section V contains an analysis of GCE momentum spectra. The momentum-space dependence of correlations between conserved charges is studied in Sec. VI. Section VII then deals with multiplicity fluctuations and correlations in limited acceptance and their extrapolation to the MCE limit. A summary is given in Sec. VIII.

\section{STATISTICAL ENSEMBLES WITH FINITE BATH}

We start out as Patriha [17] and Challa and Hetherington [26] have done but quickly take a different route.

Let us define two microcanonical partition functions, i.e., the number of microstates, for two separate systems. The first system is assumed to be enclosed in a volume $V_{1}$ and to have fixed values of extensive quantities $P_{1}^{\mu}=\left(E_{1}, P_{x, 1}, P_{y, 1}, P_{z, 1}\right)$ and $Q_{1}^{j}=\left(B_{1}, S_{1}, Q_{1}\right)$, while the second system is enclosed in a volume $V_{2}$ and has fixed values of extensive quantities $P_{2}^{\mu}=$ $\left(E_{2}, P_{x, 2}, P_{y, 2}, P_{z, 2}\right)$ and $Q_{2}^{j}=\left(B_{2}, S_{2}, Q_{2}\right)$, where $E$ is the energy of the system, $P_{x, y, z}$ are the components of its threemomentum, and $B, S$, and $Q$, are baryon number, strangeness, and electric charge, respectively. Thus we have:

$$
\begin{aligned}
& Z\left(V_{1}, P_{1}^{\mu}, Q_{1}^{j}\right)=\sum_{\left\{N_{1}^{i}\right\}} Z_{N_{1}^{i}}\left(V_{1}, P_{1}^{\mu}, Q_{1}^{j}\right), \\
& \text { and } Z\left(V_{2}, P_{2}^{\mu}, Q_{2}^{j}\right),
\end{aligned}
$$

where $Z_{N_{1}^{i}}\left(V_{1}, P_{1}^{\mu}, Q_{1}^{j}\right)$ denotes the number of microstates of system 1 with additionally fixed multiplicities $N_{1}^{i}$ of particles of species $i$. Suppose that system 1 and system 2 are subject to the following constraints:

$$
\begin{gathered}
V_{g}=V_{1}+V_{2}, \\
P_{g}^{\mu}=P_{1}^{\mu}+P_{2}^{\mu}, \\
Q_{g}^{j}=Q_{1}^{j}+Q_{2}^{j} .
\end{gathered}
$$

We can then construct the partition function $Z\left(V_{g}, P_{g}^{\mu}, Q_{g}^{j}\right)$ of the joint system as the sums over all possible charge and energy-momentum split-ups:

$$
\begin{aligned}
Z\left(V_{g}, P_{g}^{\mu}, Q_{g}^{j}\right)= & \sum_{\left\{P_{1}^{\mu}\right\}} \sum_{\left\{Q_{1}^{j}\right\}} Z\left(V_{g}-V_{1}, P_{g}^{\mu}-P_{1}^{\mu}, Q_{g}^{j}-Q_{1}^{j}\right) \\
& \times Z\left(V_{1}, P_{1}^{\mu}, Q_{1}^{j}\right) .
\end{aligned}
$$

Next we construct the distribution of extensive quantities in the subsystem $V_{1}$. This is given by the ratio of the number of all microstates consistent with a given charge and energymomentum split-up and a given set of particle multiplicities to the number of all possible configurations:

$$
\begin{aligned}
P\left(P_{1}^{\mu}, Q_{1}^{j}, N_{1}^{i}\right)= & \frac{Z\left(V_{g}-V_{1}, P_{g}^{\mu}-P_{1}^{\mu}, Q_{g}^{j}-Q_{1}^{j}\right)}{Z\left(V_{g}, P_{g}^{\mu}, Q_{g}^{j}\right)} \\
& \times Z_{N_{1}^{i}}\left(V_{1}, P_{1}^{\mu}, Q_{1}^{j}\right) .
\end{aligned}
$$

We then define the weight factor $W\left(V_{1}, P_{1}^{\mu}, Q_{1}^{j} ; V_{g}, P_{g}^{\mu}, Q_{g}^{j}\right)$ such that

$$
\begin{aligned}
P\left(P_{1}^{\mu}, Q_{1}^{j}, N_{1}^{i}\right)= & W\left(V_{1}, P_{1}^{\mu}, Q_{1}^{j} ; V_{g}, P_{g}^{\mu}, Q_{g}^{j}\right) \\
& \times Z_{N_{1}^{i}}\left(V_{1}, P_{1}^{\mu}, Q_{1}^{j}\right) .
\end{aligned}
$$

By construction, the first moment of the weight factor is equal to unity:

$$
\begin{aligned}
\langle W\rangle= & \sum_{\left\{P_{1}^{\mu}\right\}} \sum_{\left\{Q_{1}^{j}\right\}} \sum_{\left\{N_{1}^{i}\right\}} W\left(V_{1}, P_{1}^{\mu}, Q_{1}^{j} ; V_{g}, P_{g}^{\mu}, Q_{g}^{j}\right) \\
& \times Z_{N_{1}^{i}}\left(V_{1}, P_{1}^{\mu}, Q_{1}^{j}\right)=1,
\end{aligned}
$$

as the distribution is properly normalized.

The weight factor $W\left(V_{1}, P_{1}^{\mu}, Q_{1}^{j} ; V_{g}, P_{g}^{\mu}, Q_{g}^{j}\right)$ generates an ensemble with statistical properties different from the limiting cases $V_{g} \rightarrow V_{1}$ (MCE), and $V_{g} \rightarrow \infty$ (GCE). This effectively allows for extrapolation of GCE results to the MCE limit. In the thermodynamic limit ( $V_{1}$ sufficiently large) a family of thermodynamically equivalent (same densities) ensembles is generated. In principle any other (arbitrary) choice of $W\left(V_{1}, P_{1}^{\mu}, Q_{1}^{j} ; V_{g}, P_{g}^{\mu}, Q_{g}^{j}\right)$ could be taken. In this work we confine ourselves, however, to the situation discussed above. Please note that all microstates consistent with the same set of extensive quantities $\left(P_{1}^{\mu}, Q_{1}^{j}\right)$ have " a priori equal probabilities."

In the large volume limit, ensembles are equivalent in the sense that densities are the same. The ensembles defined by Eq. (7) and later on by Eq. (11) are no exception. If both $V_{1}$ and $V_{g}$ are sufficiently large, then the average densities in both systems will be the same, $Q_{g}^{j} / V_{g}$ and $P_{g}^{\mu} / V_{g}$, respectively. The system in $V_{1}$ will hence carry on average a certain fraction:

$$
\lambda \equiv V_{1} / V_{g},
$$

of the total charge $Q_{g}^{j}$ and four-momentum $P_{g}^{\mu}$, i.e.,

$$
\left\langle Q_{1}^{j}\right\rangle=\lambda Q_{g}^{j} \quad \text { and } \quad\left\langle P_{1}^{\mu}\right\rangle=\lambda P_{g}^{\mu}
$$

By varying the ratio $\lambda=V_{1} / V_{g}$, while keeping $\left\langle Q_{1}^{j}\right\rangle$ and $\left\langle P_{1}^{\mu}\right\rangle$ constant, we can thus study a class of systems with the same average charge content and four-momentum but different statistical properties. 


\section{A. Introducing the Monte Carlo weight $\mathcal{W}$}

Because Eq. (7) poses a formidable challenge, both mathematically and numerically, we write instead:

$$
\begin{aligned}
P\left(P_{1}^{\mu}, Q_{1}^{j}, N_{1}^{i}\right)= & \mathcal{W}^{P_{1}^{\mu}, Q_{1}^{j} ; P_{g}^{\mu}, Q_{g}^{j}}\left(V_{1} ; V_{g} \mid \beta, u_{\mu}, \mu_{j}\right) \\
& \times P_{\mathrm{gce}}\left(P_{1}^{\mu}, Q_{1}^{j}, N_{1}^{i} \mid \beta, u_{\mu}, \mu_{j}\right)
\end{aligned}
$$

where the distribution of extensive quantities $P_{1}^{\mu}, Q_{1}^{j}$ and particle multiplicities $N_{1}^{i}$ of a GCE system with temperature $T=\beta^{-1}$, volume $V_{1}$, chemical potentials $\mu_{j}$, and collective four-velocity $u_{\mu}$ is given by

$$
\begin{aligned}
P_{\text {gce }} & \left(P_{1}^{\mu}, Q_{1}^{j}, N_{1}^{i} \mid \beta, u_{\mu}, \mu_{j}\right) \\
& \equiv \frac{e^{-P_{1}^{\mu} u_{\mu} \beta} e^{Q_{1}^{j} \mu_{j} \beta}}{Z\left(V_{1}, \beta, u_{\mu}, \mu_{j}\right)} Z_{N_{1}^{i}}\left(V_{1}, P_{1}^{\mu}, Q_{1}^{j}\right),
\end{aligned}
$$

where $\mu_{j}=\left(\mu_{B}, \mu_{S}, \mu_{Q}\right)$, summarizes the chemical potentials associated with baryon number, strangeness, and electric charge in a vector. The normalization in Eq. (12) is given by the GCE partition function $Z\left(V_{1}, \beta, u_{\mu}, \mu_{j}\right)$, i.e., the number of all microstates averaged over the Boltzmann weights $e^{-P_{1}^{\mu} u_{\mu} \beta}$ and $e^{Q_{1}^{j} \mu_{j} \beta}$ :

$$
\begin{aligned}
Z\left(V_{1}, \beta, u_{\mu}, \mu_{j}\right)= & \sum_{\left\{P_{1}^{\mu}\right\}} \sum_{\left\{Q_{1}^{j}\right\}} \sum_{\left\{N_{1}^{i}\right\}} e^{-P_{1}^{\mu} u_{\mu} \beta} e^{Q_{1}^{j} \mu_{j} \beta} \\
& \times Z_{N_{1}^{i}}\left(V_{1}, P_{1}^{\mu}, Q_{1}^{j}\right) .
\end{aligned}
$$

The new weight factor $\mathcal{W}^{P_{1}^{\mu}, Q_{1}^{j} ; P_{g}^{\mu}, Q_{g}^{j}}\left(V_{1} ; V_{g} \mid \beta, u_{\mu}, \mu_{j}\right)$ now reads:

$$
\begin{gathered}
\mathcal{W}^{P_{1}^{\mu}, Q_{1}^{j} ; P_{g}^{\mu}, Q_{g}^{j}}\left(V_{1} ; V_{g} \mid \beta, u_{\mu}, \mu_{j}\right) \\
=Z\left(V_{1}, \beta, u_{\mu}, \mu_{j}\right) \frac{e^{-\left(P_{g}^{\mu}-P_{1}^{\mu}\right) u_{\mu} \beta} e^{\left(Q_{g}^{j}-Q_{1}^{j}\right) \mu_{j} \beta}}{e^{-P_{g}^{\mu} u_{\mu} \beta} e^{Q_{g}^{j} \mu_{j} \beta}} \\
\quad \times \frac{Z\left(V_{g}-V_{1}, P_{g}^{\mu}-P_{1}^{\mu}, Q_{g}^{j}-Q_{1}^{j}\right)}{Z\left(V_{g}, P_{g}^{\mu}, Q_{g}^{j}\right)} .
\end{gathered}
$$

In the case of an ideal (noninteracting) gas, Eq. (14) can be written [21,27] as:

$$
\begin{aligned}
& \mathcal{W}^{P_{1}^{\mu}, Q_{1}^{j} ; P_{g}^{\mu}, Q_{g}^{j}}\left(V_{1} ; V_{g} \mid \beta, u_{\mu}, \mu_{j}\right) \\
& =Z\left(V_{1}, \beta, u_{\mu}, \mu_{j}\right) \frac{\mathcal{Z}^{P_{g}^{\mu}-P_{1}^{\mu}, Q_{g}^{j}-Q_{1}^{j}}\left(V_{g}-V_{1}, \beta, u_{\mu}, \mu_{j}\right)}{\mathcal{Z}^{P_{g}^{\mu}, Q_{g}^{j}}\left(V_{g}, \beta, u_{\mu}, \mu_{j}\right)} .
\end{aligned}
$$

The advantage of Eq. (11), compared to Eq. (7), is that the distribution $P_{\text {gce }}\left(P_{1}^{\mu}, Q_{1}^{j}, N_{1}^{i} \mid \beta, u_{\mu}, \mu_{j}\right)$ can easily be sampled for Boltzmann particles, while a suitable approximation for the weight $\mathcal{W}^{P_{1}^{\mu}, Q_{1}^{j} ; P_{g}^{\mu}, Q_{g}^{j}}\left(V_{1} ; V_{g} \mid \beta, u_{\mu}, \mu_{j}\right)$ is available.

Again, by construction, the first moment of the new weight factor is equal to unity:

$$
\begin{aligned}
\langle\mathcal{W}\rangle= & \sum_{\left\{P_{1}^{\mu}\right\}} \sum_{\left\{Q_{1}^{j}\right\}} \sum_{\left\{N_{1}^{i}\right\}} \mathcal{W}^{P_{1}^{\mu}, Q_{1}^{j} ; P_{g}^{\mu}, Q_{g}^{j}}\left(V_{1} ; V_{g} \mid \beta, u_{\mu}, \mu_{j}\right) \\
& \times P_{\text {gce }}\left(P_{1}^{\mu}, Q_{1}^{j}, N_{1}^{i} \mid \beta, u_{\mu}, \mu_{j}\right)=1 .
\end{aligned}
$$

In principle, Eq. (7) and Eq. (11) are equivalent. In fact, Eq. (7) can be obtained by taking the limit $\left(\mu_{B}, \mu_{S}, \mu_{Q}\right)=$
$(0,0,0), u_{\mu}=(1,0,0,0)$, and $\beta \rightarrow 0$ of Eq. (11). However, as one can already see, $\left\langle\mathcal{W}^{n}\right\rangle \neq\left\langle W^{n}\right\rangle$. Higher, and in particular the second, moments of the weight factors $W$ and $\mathcal{W}$ are a measure of the statistical error to be expected for a finite sample of events. The larger the higher moments of the weight factor, the larger the statistical error, and the slower the convergence with sample size. Please see also Appendices A and B.

As GCE and MCE densities are the same in the system $V_{g}$, these values are effectively regulated by intensive parameters $\beta, \mu_{j}$, and $u_{\mu}$. In essence, if you want to study a system with average $\left\langle Q_{1}^{j}\right\rangle$, then sample the GCE with $\left\langle Q_{1}^{j}\right\rangle$ and calculate the weight according to Eq. (15). This will result in a low statistical error for finite samples (as shown in later sections) and allow for extrapolation to the MCE limit.

We will now first calculate the weight factor Eq. (15) and then take the appropriate limits. With the appropriate choice of $\beta, \mu_{j}$, and $u_{\mu}$ the calculation of Eq. (15) is particularly easy in the large volume limit [27].

\section{B. Calculating the Monte Carlo weight $\mathcal{W}$}

In this article, the total number of (potentially) conserved extensive quantities in a hadron resonance gas is $L=J+4=$ $3+4=7$, where $J=3$ is the number of charges $(B, S, Q)$ and there are four components of the four-momentum. Including all extensive quantities into a single vector:

$$
\mathcal{Q}^{l}=\left(Q^{j}, P^{\mu}\right)=\left(B, S, Q, E, P_{x}, P_{y}, P_{z}\right),
$$

the weight Eq. (15) can be expressed as:

$$
\begin{aligned}
& \mathcal{W}^{\mathcal{Q}_{1}^{l} ; \mathcal{Q}_{g}^{l}}\left(V_{1} ; V_{g} \mid \beta, u_{\mu}, \mu_{j}\right) \\
& =Z\left(V_{1}, \beta, u_{\mu}, \mu_{j}\right) \frac{\mathcal{Z}^{\mathcal{Q}_{g}^{l}-\mathcal{Q}_{1}^{l}}\left(V_{g}-V_{1}, \beta, u_{\mu}, \mu_{j}\right)}{\mathcal{Z}^{\mathcal{Q}_{g}^{l}}\left(V_{g}, \beta, u_{\mu}, \mu_{j}\right)} .
\end{aligned}
$$

The general expression for the partition function $\mathcal{Z}^{\mathcal{Q}^{l}}\left(V, \beta, u_{\mu}, \mu_{j}\right)$ in the large volume limit reads [27]:

$$
\begin{aligned}
& \mathcal{Z}^{\mathcal{Q}^{l}}\left(V, \beta, u_{\mu}, \mu_{j}\right) \simeq Z\left(V, \beta, u_{\mu}, \mu_{j}\right) \\
& \quad \times \frac{1}{(2 \pi V)^{L / 2} \operatorname{det} \sigma} \exp \left(-\frac{1}{2} \frac{1}{V} \xi^{l} \xi_{l}\right),
\end{aligned}
$$

where

$$
\xi^{l}=\left(\mathcal{Q}^{k}-V \kappa_{1}^{k}\right)\left(\sigma^{-1}\right)_{k}^{l}
$$

and

$$
\sigma_{k}^{l}=\left(\kappa_{2}^{1 / 2}\right)_{k}^{l} .
$$

Here $\kappa_{1}$ and $\kappa_{2}$ are the GCE vector of mean values and the GCE covariance matrix, respectively. The values of $\beta, \mu_{j}$, and $u_{\mu}$ are chosen such that

$$
\left.\frac{\partial \mathcal{Z}^{\mathcal{Q}^{l}}}{\partial \mathcal{Q}^{l}}\right|_{\mathcal{Q}^{l}=\mathcal{Q}_{e q}^{l}}=0_{l} .
$$

The approximation (19) gives then a reliable description of $\mathcal{Z}^{\mathcal{Q}_{g}^{l}}$ around the equilibrium value $\mathcal{Q}_{g}^{l}=V_{g} \kappa_{1}^{l}$, provided $V_{g}$ is sufficiently large. The charge vector, Eq. (20), is then equal to the null-vector $\xi_{l}=0_{l}\left(\mathcal{Q}_{g}^{l}=V_{g} \kappa_{1}^{l}\right)$. 
For the normalization in Eq. (18) we then find:

$$
\left.\mathcal{Z}^{\mathcal{Q}_{g}^{l}}\left(V_{g}, \beta, u_{\mu}, \mu_{j}\right)\right|_{\mathcal{Q}_{g}^{l}=\mathcal{Q}_{g, \mathrm{eq}}^{l}} \simeq \frac{Z\left(V_{g}, \beta, u_{\mu}, \mu_{j}\right)}{\left(2 \pi V_{g}\right)^{L / 2} \operatorname{det} \sigma} \exp (0) \text {. }
$$

For the numerator we obtain:

$$
\begin{aligned}
& \left.\mathcal{Z}^{\mathcal{Q}_{g}^{l}-\mathcal{Q}_{1}^{l}}\left(V_{g}-V_{1}, \beta, u_{\mu}, \mu_{j}\right)\right|_{\mathcal{Q}_{g}^{l}=\mathcal{Q}_{g, \mathrm{eq}}^{l}} \\
& \quad \simeq \frac{Z\left(V_{g}-V_{1}, \beta, u_{\mu}, \mu_{j}\right)}{\left[2 \pi\left(V_{g}-V_{1}\right)\right]^{L / 2} \operatorname{det} \sigma} \exp \left[-\frac{1}{2} \frac{1}{\left(V_{g}-V_{1}\right)} \xi^{l} \xi_{l}\right],
\end{aligned}
$$

where in Eq. (24) we write for the charge vector Eq. (20):

$$
\xi^{l}=\left(\Delta \mathcal{Q}_{2}\right)^{k}\left(\sigma^{-1}\right)_{k}^{l}
$$

Then, using $\mathcal{Q}_{g}^{k}=\mathcal{Q}_{g \text {,eq }}^{k}=V_{g} \kappa_{1}^{k}$, we find:

$$
\left(\Delta \mathcal{Q}_{2}\right)^{k}=\left(\mathcal{Q}_{g}-\mathcal{Q}_{1}\right)^{k}-\left(V_{g}-V_{1}\right) \kappa_{1}^{k}=-\left(\mathcal{Q}_{1}-V_{1} \kappa_{1}\right)^{k} .
$$

Substituting Eq. (23) and Eq. (24) into Eq. (18) yields:

$$
\begin{aligned}
&\left.\mathcal{W}^{\mathcal{Q}_{1}^{l} ; \mathcal{Q}_{g}^{l}}\left(V_{1} ; V_{g} \mid \beta, u_{\mu}, \mu_{j}\right)\right|_{\mathcal{Q}_{g}^{l}=\mathcal{Q}_{g, \text { eq }}^{l}} \simeq \frac{Z\left(V_{1}, \beta, u_{\mu}, \mu_{j}\right) Z\left(V_{g}-V_{1}, \beta, u_{\mu}, \mu_{j}\right)}{Z\left(V_{g}, \beta, u_{\mu}, \mu_{j}\right)} \\
& \quad \times \frac{\left(2 \pi V_{g}\right)^{L / 2} \operatorname{det} \sigma}{\left[2 \pi\left(V_{g}-V_{1}\right)\right]^{L / 2} \operatorname{det} \sigma} \exp \left[-\frac{1}{2} \frac{1}{\left(V_{g}-V_{1}\right)} \xi^{l} \xi_{l}\right] .
\end{aligned}
$$

The GCE partition functions are multiplicative in the sense that $Z\left(V_{1}, \beta, u_{\mu}, \mu_{j}\right) Z\left(V_{g}-V_{1}, \beta, u_{\mu}, \mu_{j}\right)=$ $Z\left(V_{g}, \beta, u_{\mu}, \mu_{j}\right)$, and thus the first term in Eq. (27) is equal to unity. Now using Eq. (9), $\lambda=V_{1} / V_{g}$, we can rewrite Eq. (27) as:

$$
\begin{aligned}
& \left.\mathcal{W}^{\mathcal{Q}_{1}^{l} ; \mathcal{Q}_{g}^{l}}\left(V_{1} ; V_{g} \mid \beta, u_{\mu}, \mu_{j}\right)\right|_{\mathcal{Q}_{g}^{l}=\mathcal{Q}_{g, \mathrm{eq}}^{l}} \\
& \simeq \frac{1}{(1-\lambda)^{L / 2}} \exp \left[-\frac{1}{2}\left(\frac{\lambda}{1-\lambda}\right) \frac{1}{V_{1}} \xi^{l} \xi_{l}\right] .
\end{aligned}
$$

Model parameters are hence the intensive variables inverse temperature $\beta$, four-velocity $u^{\mu}$, and chemical potentials $\mu^{j}$, which regulate energy and charge densities and collective motion. Provided $V_{1}$ is sufficiently large, we have defined a family of thermodynamically equivalent ensembles, which can now be studied in their dependence of fluctuation and correlation observables on the size of the bath $V_{2}=V_{g}-V_{1}$. Hence, we can test the sensitivity of such observables, for example, to globally applied conservation laws. The expectation values $\langle\ldots\rangle$ are then identical to GCE expectation values, while higher moments will depend crucially on the choice of $\lambda$.

\section{The limits of $\mathcal{W}$}

The largest weight is given to states for which $\xi^{l} \xi_{l}=0$, i.e., with extensive quantities $\mathcal{Q}_{1}^{l}=\mathcal{Q}_{1, \text { eq. }}^{l}$. Hence, the maximal weight a microstate (or event) at a given value of $\lambda=V_{1} / V_{g}$ can assume is $\mathcal{W}_{\max }^{\mathcal{Q}_{1}^{l} ; \mathcal{Q}_{g}^{l}}\left(V_{1} ; V_{g} \mid \beta, u_{\mu}, \mu_{j}\right)=(1-\lambda)^{-L / 2}$. Taking the limits of Eq. (28), it is easy to see that:

$$
\lim _{\lambda \rightarrow 0} \mathcal{W}^{\mathcal{Q}_{1}^{l} ; \mathcal{Q}_{g}^{l}}\left(V_{1} ; V_{g} \mid \beta, u_{\mu}, \mu_{j}\right)=1,
$$

i.e., for $\lambda=0$ we sample the GCE, and all events have a weight equal to unity. Hence, we also find $\left\langle\mathcal{W}^{2}\right\rangle=1$ and therefore $\left\langle(\Delta \mathcal{W})^{2}\right\rangle=0$, implying a low statistical error. For $\lambda \rightarrow 1$, we effectively approach a "sample-reject" procedure, as (for instance) used in Refs. [19,20], and

$$
\lim _{\lambda \rightarrow 1} \mathcal{W}^{\mathcal{Q}_{1}^{l} ; \mathcal{Q}_{g}^{l}}\left(V_{1} ; V_{g} \mid \beta, u_{\mu}, \mu_{j}\right) \propto \delta\left(\mathcal{Q}_{1}^{l}-V_{1} \kappa_{1}^{l}\right) .
$$

However, as now not all events have equal weight, $\left\langle(\Delta \mathcal{W})^{2}\right\rangle$ grows and so too does the statistical error of finite samples. Moreover, the larger the number $L$ of extensive quantities considered for reweighting, the larger the statistical uncertainty.

\section{THE GCE SAMPLING PROCEDURE}

The Monte Carlo sampling procedure for a GCE system in the Boltzmann approximation is now explained. The system to be sampled is assumed to be in an equilibrium state enclosed in a volume $V_{1}$ with temperature $T=\beta^{-1}$ and chemical potentials $\mu_{j}=\left(\mu_{B}, \mu_{S}, \mu_{Q}\right)$. Additionally, the system is assumed to be at rest. The four-velocity is then $u^{\mu}=(1,0,0,0)$ and the four-temperature is $\beta^{\mu}=(\beta, 0,0,0)$. In this case, multiplicity distributions are Poissonian, while momentum spectra are of Boltzmann type.

The GCE sampling process is composed of four steps, each discussed below.

\section{A. Multiplicity generation}

In the first step, we randomly sample multiplicities $N_{1}^{i}$ of all particle species $i$ considered in the model. The expectation value of the multiplicity of thermal Boltzmann particles in the GCE is given by

$$
\left\langle N_{1}^{i}\right\rangle=\frac{g_{i} V_{1}}{2 \pi^{2}} m_{i}^{2} T K_{2}\left(\frac{m_{i}}{T}\right) e^{\mu_{i} / T} .
$$

Multiplicities $\left\{N_{1}^{i}\right\}_{n}$ are randomly generated for each event $n$ according to Poissonians with mean values $\left\langle N_{1}^{i}\right\rangle$ :

$$
P\left(N_{1}^{i}\right)=\frac{\left\langle N_{1}^{i}\right\rangle^{N_{1}^{i}}}{N_{1}^{i} !} e^{-\left\langle N_{1}^{i}\right\rangle} .
$$

In the above, $m_{i}$ and $g_{i}$ are the mass and degeneracy factor of a particle of species $i$, respectively. The chemical potential $\mu_{i}=\mu_{j} q_{i}^{j}=\mu_{B} b_{i}+\mu_{S} s_{i}+\mu_{Q} q_{i}$, where $q_{i}^{j}=\left(b_{i}, s_{i}, q_{i}\right)$ represents the quantum number content of a particle of species $i$.

\section{B. Momentum spectra}

In the second step, we generate momenta for each particle according to a Boltzmann spectrum. For a static thermal source 
spherical coordinates are convenient:

$$
\frac{d N_{i}}{d|p|}=\frac{g_{i} V_{1}}{2 \pi^{2}} T^{3}|p|^{2} e^{-\varepsilon / T} .
$$

These momenta are then isotropically distributed in momentum space. Hence:

$$
\begin{aligned}
p_{x} & =|p| \sin \theta \cos \phi, \\
p_{y} & =|p| \sin \theta \sin \phi, \\
p_{z} & =|p| \cos \theta, \\
\varepsilon & =\sqrt{|p|^{2}+m^{2}},
\end{aligned}
$$

where $p_{x}, p_{y}$, and $p_{z}$ are the components of the threemomentum, $\varepsilon$ is the energy, and $|p|=\sqrt{p_{x}^{2}+p_{y}^{2}+p_{z}^{2}}$ is the total momentum. The polar and azimuthal angles are sampled according to

$$
\begin{aligned}
& \theta=\cos ^{-1}[2(x-0.5)], \\
& \phi=2 \pi(x-0.5),
\end{aligned}
$$

where $x$ is uniformly distributed between 0 and 1. Additionally, we calculate the transverse momentum $p_{T}$ and rapidity $y$ for each particle:

$$
\begin{aligned}
p_{T} & =\sqrt{p_{x}^{2}+p_{y}^{2}}, \\
y & =\frac{1}{2} \ln \left(\frac{\varepsilon+p_{z}}{\varepsilon-p_{z}}\right) .
\end{aligned}
$$

Finally, we distribute particles homogeneously in a sphere of radius $r_{1}$ and calculate decay times based on the Breit-Wigner width of the resonances.

\section{Resonance decay}

The third step (if applicable) is resonance decay. We follow the prescription used by the authors of the THERMINATOR package [28], and perform only two- and three-body decays, while allowing for successive decay of unstable daughter particles. Only strong decays are considered, while weak and electromagnetic decays are omitted. Particle decay is first calculated in the parent's rest frame, with daughter momenta then boosted into the laboratory frame. Finally, decay positions are generated based on the parent's production point, momentum, and lifetime.

Throughout this article, always only the lightest states of the following baryons:

$$
\begin{array}{llllllll}
\mathrm{p} & \mathrm{n} & \Lambda & \Sigma^{+} & \Sigma^{-} & \Xi^{-} & \Xi^{0} & \Omega^{-}
\end{array}
$$

and mesons:

$$
\pi^{+} \quad \pi^{-} \pi^{0} \quad K^{+} K^{-} \quad K^{0}
$$

are considered as stable. The system could now be given collective velocity $u^{\mu}$.

\section{Reweighting}

In the fourth step, we calculate the values of extensive quantities for the events generated by iterating over the particle list of each event. For the values of extensive quantities $\mathcal{Q}_{1, n}^{l}=$ $\left(B_{1, n}, S_{1, n}, Q_{1, n}, E_{1, n}, P_{x, 1, n}, P_{y, 1, n}, P_{z, 1, n}\right)$ in subsystem $V_{1}$ of event $n$ we write:

$$
\mathcal{Q}_{1, n}^{l}=\sum_{\text {particles } i_{n}} \mathfrak{q}_{i_{n}}^{l}
$$

where $\mathfrak{q}_{i_{n}}^{l}=\left(b_{i_{n}}, s_{i_{n}}, q_{i_{n}}, \varepsilon_{i_{n}}, p_{x, i_{n}}, p_{y, i_{n}}, p_{z, i_{n}}\right)$ is the "charge vector" of particle $i$ in event $n$. Based on $\mathcal{Q}_{1, n}^{l}$ we calculate the weight $w_{n}$ for the event:

$$
w_{n}=\mathcal{W}^{\mathcal{Q}_{1, n}^{l} ; \mathcal{Q}_{g}^{l}}\left(V_{1} ; V_{g} \mid \beta, u_{\mu}, \mu_{j}\right),
$$

according to Eq. (28). Please note that all microstates with the same set of extensive quantities $\mathcal{Q}_{1, n}^{l}$ are still counted equally.

\section{EXTRAPOLATING FULLY PHASE SPACE INTEGRATED QUANTITIES TO THE MCE}

We now attempt to extrapolate fully phase space integrated grand canonical results to the microcanonical limit. For this we iteratively generate, reweight, and analyze samples of events for various values of $\lambda=V_{1} / V_{g}$. By construction of the weight factor $\mathcal{W}$, Eq. (28), we extrapolate in a systematic fashion such that, for instance, particle momentum spectra as well as mean values of extensive quantities remain unchanged. However, all variances and covariances of extensive quantities subject to reweighting converge linearly to their microcanonical values.

This can be seen from the form of the analytical approximation to the grand canonical distribution of (fully phase space integrated) extensive quantities $P_{\text {gce }}\left(\mathcal{Q}_{1}^{l}\right)$ [from Eq. (19)]:

$$
P_{\text {gce }}\left(\mathcal{Q}_{1}^{l}\right) \simeq \frac{1}{\left(2 \pi V_{1}\right)^{L / 2} \operatorname{det} \sigma} \exp \left(-\frac{1}{2} \frac{1}{V_{1}} \xi^{l} \xi_{l}\right),
$$

where the variable $\xi^{l}$ is given by Eq. (20). Now taking the weight factor $\mathcal{W}_{\lambda}$, Eq. (28) ( $\sigma$ and $\xi_{l}$ are the same in both equations), we obtain for the distribution $P_{\lambda}\left(\mathcal{Q}_{1}^{l}\right)$ of extensive quantities $\mathcal{Q}_{1}^{l}$ in subsystem 1:

$$
\begin{aligned}
P_{\lambda}\left(\mathcal{Q}_{1}^{l}\right) & \simeq \mathcal{W}_{\lambda}^{\mathcal{Q}_{1}^{l} ; \mathcal{Q}_{g}^{l}} P_{\text {gce }}\left(\mathcal{Q}_{1}^{l}\right) \\
& \simeq \frac{1}{\left[2 \pi(1-\lambda) V_{1}\right]^{L / 2} \operatorname{det} \sigma} \exp \left[-\frac{1}{2} \frac{1}{(1-\lambda) V_{1}} \xi^{l} \xi_{l}\right] .
\end{aligned}
$$

This is essentially the same multivariate normal distribution as the grand canonical version $P_{\text {gce }}\left(\mathcal{Q}_{1}^{l}\right)$, however linearly contracted. We will compare Monte Carlo results to Eq. (48).

The Monte Carlo output is essentially a distribution $P_{\mathrm{MC}}\left(X_{1}, X_{2}, X_{3}, \ldots\right)$ of a set of observables $X_{1}, X_{2}, X_{3}$, etc.. For all practical purposes this distribution is obtained by histograming all events $n$ according to their values of $X_{1, n}, X_{2, n}, X_{3, n}$, etc., and their weight $w_{n}$. One can then define moments of two observables $X_{i}$ and $X_{j}$ through:

$$
\left\langle X_{i}^{n} X_{j}^{m}\right\rangle \equiv \sum_{X_{i}, X_{j}} X_{i}^{n} X_{j}^{m} P_{M C}\left(X_{i}, X_{j}\right) .
$$


Additionally, we define the variance $\left\langle\left(\Delta X_{i}\right)^{2}\right\rangle$ and the covariance $\left\langle\Delta X_{i} \Delta X_{j}\right\rangle$, respectively, as

$$
\begin{aligned}
\left\langle\left(\Delta X_{i}\right)^{2}\right\rangle & \equiv\left\langle X_{i}^{2}\right\rangle-\left\langle X_{i}\right\rangle^{2}, \quad \text { and } \\
\left\langle\Delta X_{i} \Delta X_{j}\right\rangle & \equiv\left\langle X_{i} X_{j}\right\rangle-\left\langle X_{i}\right\rangle\left\langle X_{j}\right\rangle .
\end{aligned}
$$

In the following, we use the scaled variance $\omega_{i}$ and the correlation coefficient $\rho_{i j}$ defined as

$$
\begin{aligned}
\omega_{i} & \equiv \frac{\left\langle\left(\Delta X_{i}\right)^{2}\right\rangle}{\left\langle X_{i}\right\rangle} \quad \text { and } \\
\rho_{i j} & \equiv \frac{\left\langle\Delta X_{i} \Delta X_{j}\right\rangle}{\sqrt{\left\langle\left(\Delta X_{i}\right)^{2}\right\rangle\left\langle\left(\Delta X_{j}\right)^{2}\right\rangle}} .
\end{aligned}
$$

Let us consider a static and neutral system with fourvelocity $u_{\mu}=(1,0,0,0)$, chemical potentials $\mu_{j}=(0,0,0)$, local temperature $T=\beta^{-1}=0.160 \mathrm{GeV}$, and volume $V_{1}=$ $2000 \mathrm{fm}^{3}$. This is a system large enough ${ }^{3}$ to use the large volume approximation worked out in Sec. II.

In Figs. 1 and 2 we show the results of Monte Carlo runs of $2.5 \times 10^{4}$ events each. Each value of $\lambda$ has been sampled 20 times to allow for calculation of a statistical uncertainty estimate. Nineteen different values of $\lambda$ have been studied. In this case study, the extensive quantities baryon number $B$, strangeness $S$, electric charge $Q$, energy $E$, and longitudinal momentum $P_{z}$ are considered for reweighting. Conservation of transverse momenta $P_{x}$ and $P_{y}$ can be shown not to affect the $\Delta p_{T, i}$ and $\Delta y_{i}$ dependence of multiplicity fluctuations and correlations studied in the following sections. Their $\Delta y_{i}$ dependence is, however, rather sensitive to $P_{z}$ conservation. Angular correlations (not studied in this article), however, are strongly sensitive to joint $P_{x}$ and $P_{y}$ conservation [21,22].

In Fig. 1 (top) we show the results for mean values of baryon number $\langle B\rangle$, strangeness $\langle S\rangle$, electric charge $\langle Q\rangle$, energy $\langle E\rangle$, and the momenta $\left\langle P_{x}\right\rangle$ and $\left\langle P_{z}\right\rangle$. The solid lines represent GCE values. Only the expectation value of energy is not equal to 0 , as the system sampled is assumed to be static and neutral with $T \neq 0$. The evolution of the respective variances is shown in Fig. 1 (bottom). Variances of extensive quantities subject to reweighting converge linearly to 0 as $\lambda$ goes to 1 . One notes that $\left\langle\left(\Delta P_{x}\right)^{2}\right\rangle$ remains constant (within error bars), as this quantity is not reweighted in this case study. Please note that on many data points the error bars are smaller than the symbol used.

In Fig. 2 (top) we show the evolution of covariances $\langle\Delta B \Delta S\rangle,\langle\Delta B \Delta Q\rangle,\langle\Delta S \Delta Q\rangle$, and $\langle\Delta E \Delta Q\rangle$ with the "size of the bath." As seen, the covariances between quantities considered for reweighting also converge linearly to 0 . In a neutral system, covariances between energy and charge are equal to 0 . As an example, we show $\langle\Delta E \Delta Q\rangle$. In a static system, also the covariances between momenta and any other extensive quantity are equal to 0 . As an example, we show $\left\langle\Delta E \Delta P_{z}\right\rangle$. The correlation coefficients, Eq. (53), however,

\footnotetext{
${ }^{3}$ Generally it is not easy to say when a system is "large enough" for the large volume approximation to be valid. Here we find good agreement with asymptotic analytic solutions. Charged systems, or Bose-Einstein/Fermi-Dirac systems, usually converge more slowly to their asymptotic solution.
}
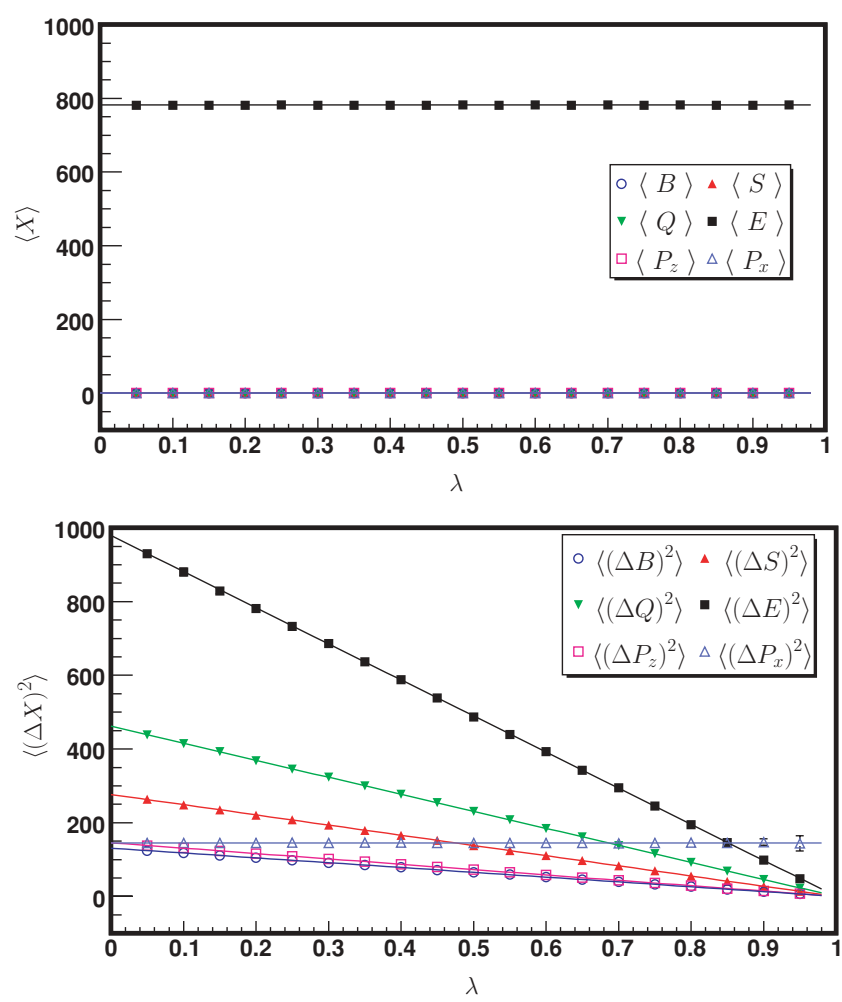

FIG. 1. (Color online) Mean values (top) and variances (bottom) of various extensive quantities, as listed in the legends, as a function of $\lambda$. Each marker and its error bar represents the result of 20 Monte Carlo runs of $2.5 \times 10^{4}$ events each. Nineteen different equally spaced values of $\lambda$ have been investigated. Solid lines indicate GCE values (top) or linear extrapolations from the GCE value to the MCE limit (bottom).

remain constant as a function of $\lambda$, as shown in Fig. 2 (bottom). The values of fully phase space integrated correlation coefficients $\rho_{B S}, \rho_{B Q}$, and $\rho_{S Q}$ can be compared to the GCE results denoted by the solid lines shown in Figs. 5-7 in Sec. VI.

The variances and covariances converge linearly from their GCE values to their respective MCE limits in the large volume limit. The dependence of $\left\langle\left(\Delta X_{i}\right)^{2}\right\rangle$, Eq. (50), and $\left\langle\Delta X_{i} \Delta X_{j}\right\rangle$, Eq. (51), on the size of the bath $\lambda$ is given by:

$$
\begin{aligned}
\left\langle\left(\Delta X_{i}\right)^{2}\right\rangle_{\lambda} & =(1-\lambda)\left\langle\left(\Delta X_{i}\right)^{2}\right\rangle_{\text {gce }}+\lambda\left\langle\left(\Delta X_{i}\right)^{2}\right\rangle_{\text {mce }} \\
\left\langle\Delta X_{i} \Delta X_{j}\right\rangle_{\lambda} & =(1-\lambda)\left\langle\Delta X_{i} \Delta X_{j}\right\rangle_{\text {gce }}+\lambda\left\langle\Delta X_{i} \Delta X_{j}\right\rangle_{\text {mce }} .
\end{aligned}
$$

Mean values $\left\langle X_{i}\right\rangle_{\lambda}$ remain constant. This implies that the scaled variance $\omega$ of multiplicity fluctuations, Eq. (52), also converges linearly:

$$
\omega_{\lambda} \equiv \frac{\left\langle\left(\Delta N_{i}\right)^{2}\right\rangle_{\lambda}}{\left\langle N_{i}\right\rangle_{\lambda}}=(1-\lambda) \omega_{\mathrm{gce}}+\lambda \omega_{\mathrm{mce}},
$$

from its GCE value $\omega_{\text {gce }}$ to the MCE limit $\omega_{\text {mce }}$. Please note that Eqs. (54)-(56) are equivalent to the "acceptance scaling" approximation ${ }^{4}$ used in Refs. [29-31]. For the correlation

\footnotetext{
${ }^{4}$ For the situation discussed here one could equivalently say that particles are randomly drawn from coordinate space of the total
} 

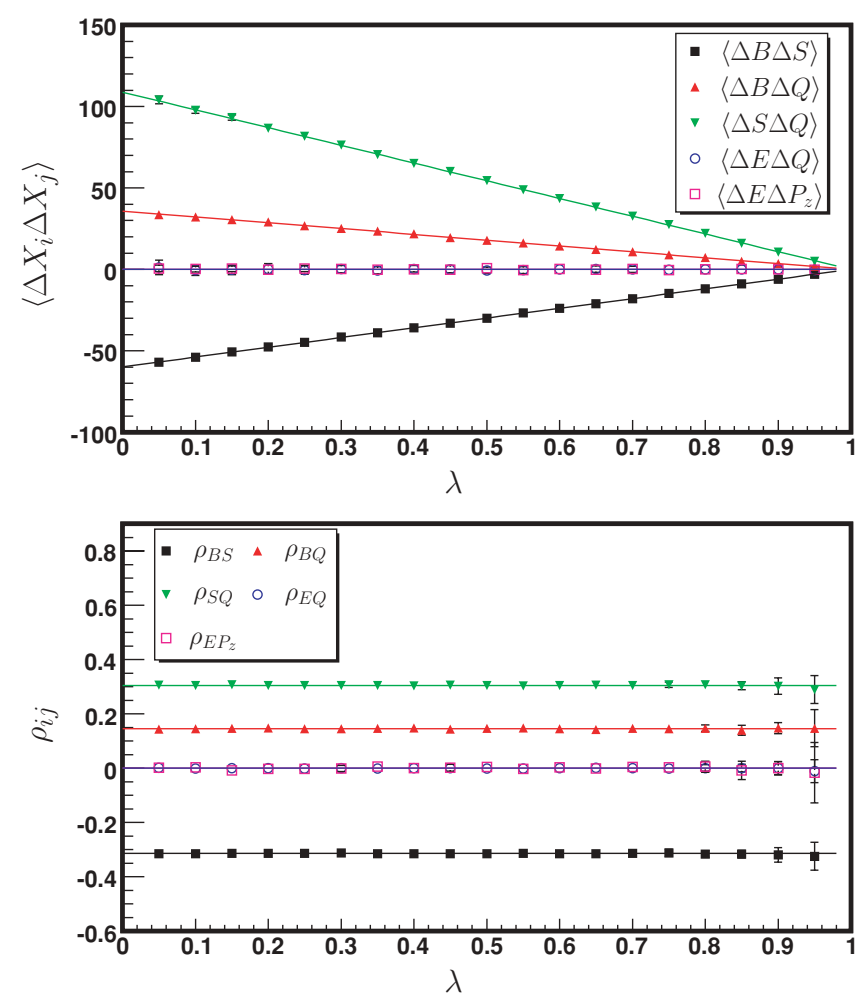

FIG. 2. (Color online) Covariances (top) and correlation coefficients (bottom) between various extensive quantities, as listed in the legends, as a function of $\lambda$. Solid lines indicate linear extrapolations from the GCE value to the MCE limit (top) or GCE values (bottom); the rest as in Fig. 1.

coefficient, Eq. (53),

$$
\rho_{\lambda} \equiv \frac{\left\langle\Delta X_{i} \Delta X_{j}\right\rangle_{\lambda}}{\sqrt{\left\langle\left(\Delta X_{i}\right)^{2}\right\rangle_{\lambda}\left\langle\left(\Delta X_{j}\right)^{2}\right\rangle_{\lambda}}},
$$

the story is more complicated. In case both $X_{i}$ and $X_{j}$ are reweighted and measured in full phase space, we find:

$$
\left\langle\left(\Delta X_{i}\right)^{2}\right\rangle_{\mathrm{mce}}=\left\langle\left(\Delta X_{j}\right)^{2}\right\rangle_{\mathrm{mce}}=\left\langle\Delta X_{i} \Delta X_{j}\right\rangle_{\mathrm{mce}}=0,
$$

and the correlation coefficient $\rho_{\lambda}$, Eq. (57), is independent of the value of $\lambda$, see Fig. 2 . In all other cases, one needs to extrapolate Eqs. (54) and (55) separately and then calculate the correlation coefficient.

We have therefore successively transformed our Monte Carlo sample. As $\lambda \rightarrow 1$, we give larger and larger weight to events in the immediate vicinity of the equilibrium expectation value and smaller and smaller weight to events away from it. The distribution of extensive quantities considered for reweighting (a multivariate normal distribution in the GCE in the large volume limit) hence gets contracted to a $\delta$ function with vanishing variances and covariances, i.e., we successively highlight the properties of events that have very similar values of extensive quantities. This will have a bearing on charge

volume $V_{g}$. For the derivation of the acceptance scaling formula [29] it was, however, assumed that particles are randomly drawn from a sample in momentum space.

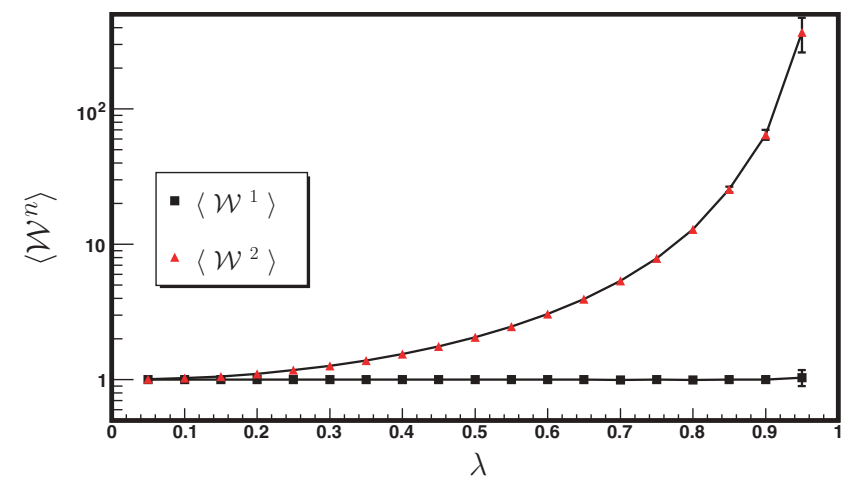

FIG. 3. (Color online) First and second moment of the weight factor Eq. (28) as a function of $\lambda$. The rest as in Fig. 1.

correlations and, in particular, multiplicity fluctuations and correlations discussed in the following sections.

The price we pay is that, as $\lambda$ grows, so too does the statistical uncertainty. In the limit $\lambda \rightarrow 1$, we approach a sample-reject type of formalism. We cannot, therefore, directly obtain the microcanonical limit for the large system size studied here, as this is prohibited by available computing power. On the bright side, however, we can extrapolate to this limit. In Fig. 3 we show the second moment of the weight factor, Eq. (28), as a function of $\lambda$. A large second moment $\left\langle\mathcal{W}^{2}\right\rangle$ implies a large statistical uncertainty and, hence, usually requires a larger sample. We mention in this context that the intermediate ensembles, between the limits of GCE and MCE, may also be of phenomenological interest.

\section{MOMENTUM SPECTRA}

We next consider momentum spectra. In Fig. 4 we show transverse momentum and rapidity spectra of positively charged hadrons, both primordial and final state, for a static thermal system.

Based on these momentum spectra we construct acceptance bins $\Delta p_{T, i}$ and $\Delta y_{i}$, as in Refs. [21-23] and [32,33]. Momentum bins are constructed such that each of the five bins constructed contains on average one fifth of the total yield of positively charged particles. The values defining the bounds of the momentum-space bins $\Delta p_{T, i}$ and $\Delta y_{i}$ are summarized in Table I.

Resonance decay shifts the transverse momentum distribution to lower average transverse momentum $\left\langle p_{T}\right\rangle$ and widens the rapidity distribution of thermal "fireballs" [34]. Final state transverse momentum bins are, hence, slightly "contracted," while final state rapidity bins get slightly "wider," when compared to their respective primordial counterparts.

Resonance decay combined with transverse as well as longitudinal flow is believed to provide a rather good description of experimentally observed momentum spectra in relativistic heavy-ion collisions at SPS and RHIC energies [28,35,36]. Our spectra, however, contain no flow and our results thus cannot be directly compared to experimental data or transport simulations. However, qualitatively one might observe effects of the kind discussed in the following. 
TABLE I. Transverse momentum and rapidity bins $\Delta p_{T, i}=\left[p_{T, i}, p_{T, i+1}\right]$ and $\Delta y_{i}=\left[y_{i}, y_{i+1}\right]$, both primordial and final state, for a static neutral Boltzmann system with temperature $T=0.160 \mathrm{GeV}$.

\begin{tabular}{lcccccc}
\hline \hline & $p_{T, 1}(\mathrm{GeV})$ & $p_{T, 2}(\mathrm{GeV})$ & $p_{T, 3}(\mathrm{GeV})$ & $p_{T, 4}(\mathrm{GeV})$ & $p_{T, 5}(\mathrm{GeV})$ & $p_{T, 6}(\mathrm{GeV})$ \\
\hline Primordial & 0.0 & 0.22795 & 0.36475 & 0.51825 & 0.73995 & 5.0 \\
Final state & 0.0 & 0.17105 & 0.27215 & 0.38785 & 0.56245 & 5.0 \\
& $y_{1}$ & $y_{2}$ & $y_{3}$ & $y_{4}$ & $y_{5}$ & $y_{6}$ \\
Primordial & -5.0 & -0.4275 & -0.1241 & 0.1241 & 0.4273 & 5.0 \\
Final state & -5.0 & -0.5289 & -0.1553 & 0.1551 & 0.5289 & 5.0 \\
\hline \hline
\end{tabular}

\section{THE MOMENTUM-SPACE DEPENDENCE OF CORRELATIONS BETWEEN CONSERVED CHARGES}

An interesting example of quantities for which the measured value depends on the observed part of the momentum spectrum are the correlation coefficients between the charges baryon number $B$, strangeness $S$, and electric charge $Q$. Please note that also the variances and covariances of the baryon number, strangeness, and electric charge distribution are sensitive to the acceptance cuts applied. Their values are additionally rather sensitive to the effects of globally enforced conservation laws. If the size of the "bath" is reduced, a change in one interval of phase space will have to be balanced (preferably) by a change in another interval and not by the "bath."
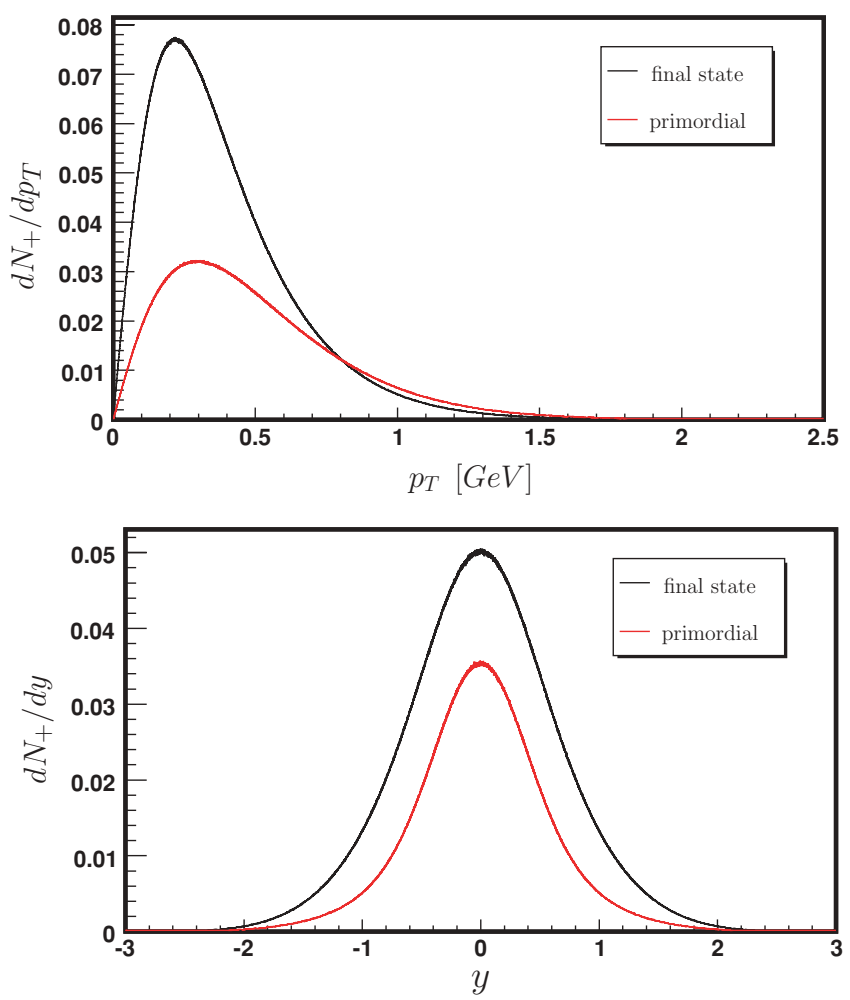

FIG. 4. (Color online) (Top) Transverse momentum spectrum of positively charged hadrons, both primordial and final state. (Bottom) Rapidity spectrum of positively charged hadrons, both primordial and final state. The number of events that have been sampled is $2 \times 10^{6}$.

\section{A. Grand canonical ensemble}

We will now consider the correlation coefficients $\rho_{B S}, \rho_{B Q}$, and $\rho_{S Q}$ in limited acceptance bins $\Delta p_{T, i}$ and $\Delta y_{i}$, as defined in Table I in the grand canonical ensemble. Particles in one momentum bin are then essentially sampled independently from particles in any other momentum-space segment, due to the "infinite bath" assumption. Nevertheless, the way in which quantum numbers are correlated is different in different momentum bins, as different particle species have, due to their different masses, different momentum spectra.

Let us first make some basic observations about the hadron resonance gas and the way in which quantum numbers are correlated in a GCE. Charge fluctuations directly probe the degrees of freedom of a system, i.e., they are sensitive to its particle mass spectrum (and its quantum number configurations). We first consider the contribution of different particle species to the covariance $\left\langle\Delta X_{i} \Delta X_{j}\right\rangle$, Eq. (51), and hence to the correlation coefficient $\rho_{i j}$, Eq. (53).

All baryons have baryon number $b=+1$. Baryons can only carry strange quarks, i.e., their strangeness is always $s \leqslant 0$. Antibaryons have $b=-1$ and $s \geqslant 0$. Hence, both groups contribute negatively to the baryon-strangeness covariance, and so $\langle\Delta B \Delta S\rangle<0$, and therefore $\rho_{B S}<0$, as indicated by the solid lines in Fig. 5.

Positively charged baryons and their antiparticles contribute positively to the baryon-electric charge covariance $\langle\Delta B \Delta Q\rangle$, while negatively charged baryons (and their anti-particles) contribute negatively. Two observations can be made on the hadron resonance gas mass spectrum: there are more positively charged baryons than negatively charged ones, and their average mass is lower, i.e., in a neutral gas $\left(\mu_{B}=\mu_{Q}=\mu_{S}=0\right)$ the contribution of positively charged baryons dominates and therefore $\langle\Delta B \Delta Q\rangle>0$ and $\rho_{B} Q>0$, as indicated by the solid lines in Fig. 6.

Mesons and their antiparticles always contribute positively to the strangeness-electric charge correlation coefficient $\rho_{S Q}$. Electrically charged strange mesons are either composed of a $u$ quark and an $\bar{s}$ quark or of a $\bar{u}$ quark and an $s$ quark (and superpositions thereof). Their contribution to $\langle\Delta S \Delta Q\rangle$ is in either case positive. On the baryonic side, only the $\Sigma^{+}$(as well as its degenerate states and their respective antiparticles) has a negative contribution to $\langle\Delta S \Delta Q\rangle$, while all other strangeness carrying baryons have either electric charge $q=-1$, or $q=0$. Therefore, we find $\rho_{S Q}>0$, as indicated by the solid lines in Fig. 7. 

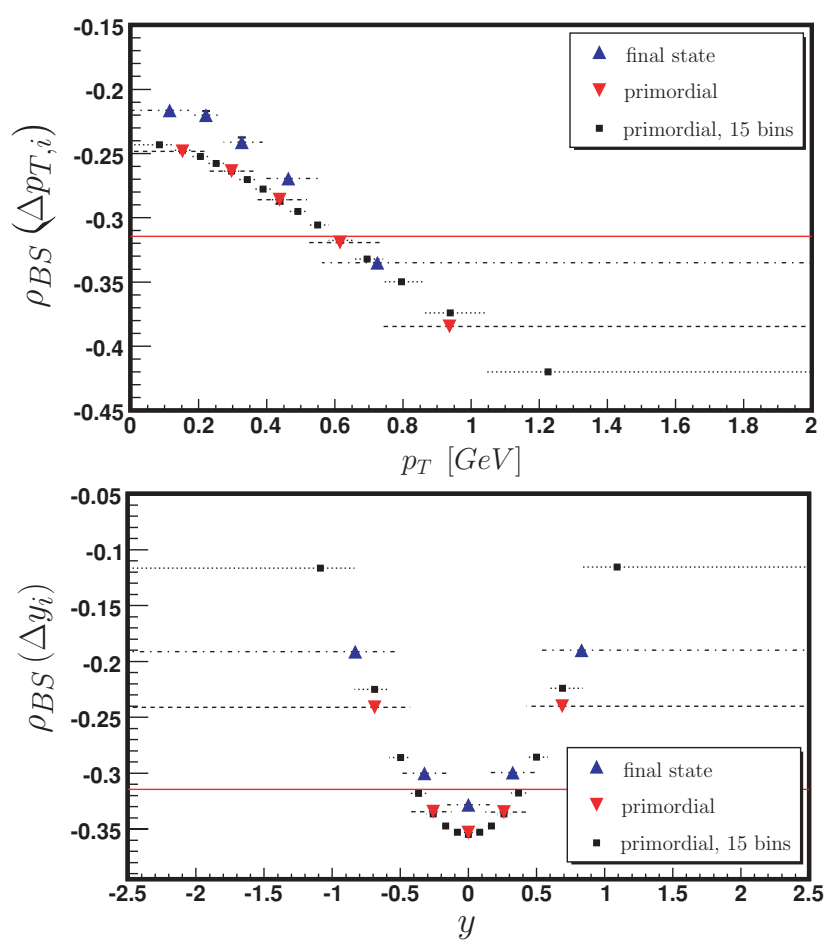

FIG. 5. (Color online) Baryon-strangeness correlation coefficient $\rho_{B S}$ in the GCE in limited acceptance windows, both primordial and final state. (Top) Transverse momentum bins $\Delta p_{T, i}$. (Bottom) Rapidity bins $\Delta y_{i}$. Horizontal error bars indicate the width and position of the momentum bins (and not an uncertainty!). Vertical error bars indicate the statistical uncertainty of 20 Monte Carlo runs of $10^{5}$ events each. The marker indicates the center of gravity of the corresponding bin. The solid lines show the fully phase space integrated GCE result.

In Figs. 5-7 we show the correlation coefficients $\rho_{B S}$ (baryon number-strangeness), $\rho_{B Q}$ (baryon number-electric charge), and $\rho_{S Q}$ (strangeness-electric charge) as measured in the acceptance bins $\Delta p_{T, i}$ and $\Delta y_{i}$ defined in Table I, both primordial and final state. The average baryon number, strangeness, and electric charge in each bin is equal to zero, as the system is assumed to be neutral. The analytical primordial values (15 bins) shown in Figs. 5-7 are calculated using analytical spectra. Please note that, again, on many data points the error bars are smaller than the symbol used.

In Tables II-IV we summarize the transverse momentum and rapidity dependence of the correlation coefficients $\rho_{B S}, \rho_{B Q}$, and $\rho_{S Q}$. The statistical error quoted corresponds to 20 Monte Carlo runs of $10^{5}$ events each. The analytical values (five bins) listed in the tables are calculated using the momentum bins defined in Table I. Mild differences between Monte Carlo and analytical results are unavoidable. The analytical values are also not exactly symmetric in $\Delta y_{i}$, as the exact size of the acceptance bins constructed is sensitive to the number of bins used for the calculation of the momentum spectra. The values of the correlation coefficient $\rho$ are also rather sensitive to exact bin size, and the fourth digit becomes somewhat unreliable.

We next attempt to explain, in turn, the rapidity dependence of $\rho_{B S}, \rho_{B Q}$, and $\rho_{S Q}$. Strange baryons are, on average,
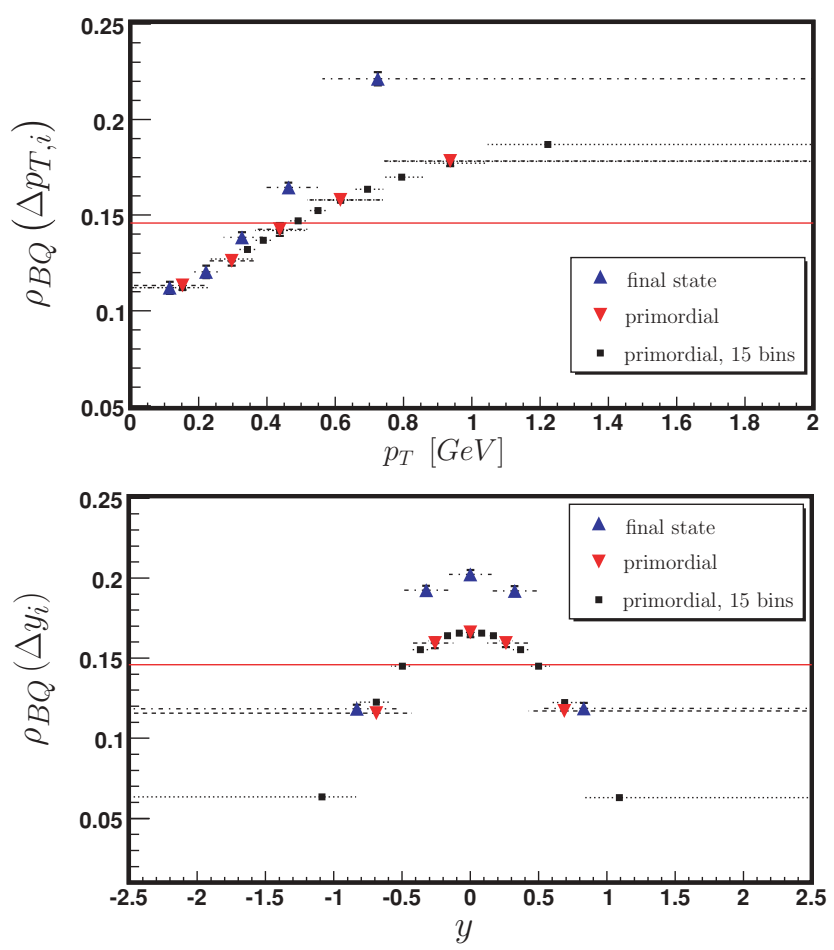

FIG. 6. (Color online) Baryon-electric charge correlation coefficient $\rho_{B Q}$ in the GCE in limited acceptance windows, both primordial and final state. (Top) Transverse momentum bins $\Delta p_{T, i}$. (Bottom) Rapidity bins $\Delta y_{i}$; the rest as in Fig. 5.
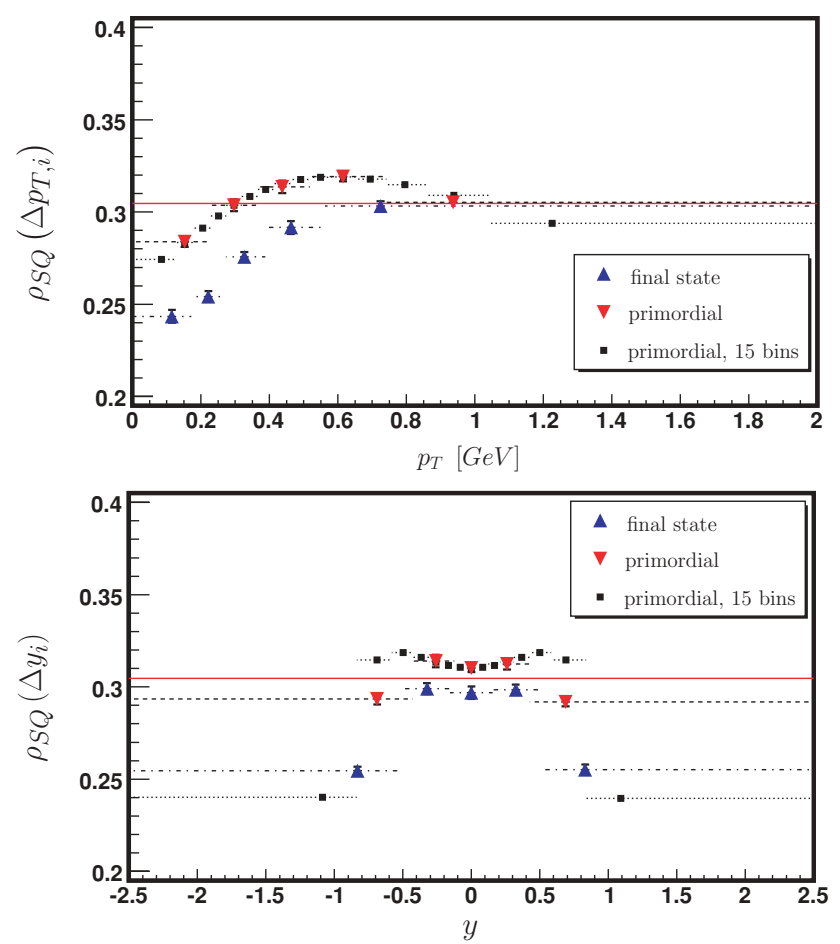

FIG. 7. (Color online) Strangeness-electric charge correlation coefficient $\rho_{S Q}$ in the GCE in limited acceptance windows, both primordial and final state. (Top) Transverse momentum bins $\Delta p_{T, i}$. (Bottom) Rapidity bins $\Delta y_{i}$; the rest as in Fig. 5 . 
TABLE II. Baryon-strangeness correlation coefficient $\rho_{B S}$ in the GCE in transverse momentum bins $\Delta p_{T, i}$ and rapidity bins $\Delta y_{i}$, both primordial and final state. For comparison, analytical values $\rho_{\text {prim }}^{\text {calc }}$ for primordial correlations are included. The statistical uncertainty corresponds to 20 Monte Carlo runs of $10^{5}$ events each.

\begin{tabular}{lccccc}
\hline \hline$\rho_{B S}$ & $\Delta p_{T, 1}$ & $\Delta p_{T, 2}$ & $\Delta p_{T, 3}$ & $\Delta p_{T, 4}$ & $\Delta p_{T, 5}$ \\
\hline$\rho_{\text {prim }}^{\text {calc }}$ & -0.2479 & -0.2641 & -0.2864 & -0.3188 & -0.3839 \\
$\rho_{\text {prim }}$ & $-0.248 \pm 0.003$ & $-0.264 \pm 0.003$ & $-0.286 \pm 0.003$ & $-0.319 \pm 0.002$ & $-0.385 \pm 0.002$ \\
$\rho_{\text {final }}$ & $-0.216 \pm 0.002$ & $-0.220 \pm 0.003$ & $-0.241 \pm 0.004$ & $-0.269 \pm 0.003$ & $-0.335 \pm 0.003$ \\
$\rho_{B S}$ & $\Delta y_{1}$ & $\Delta y_{2}$ & $\Delta y_{3}$ & $\Delta y_{4}$ & $\Delta y_{5}$ \\
$\rho_{\text {prim }}^{\text {calc }}$ & -0.2407 & -0.3345 & -0.3536 & -0.3345 & -0.2408 \\
$\rho_{\text {prim }}$ & $-0.241 \pm 0.003$ & $-0.334 \pm 0.003$ & $-0.353 \pm 0.003$ & $-0.335 \pm 0.003$ & $-0.240 \pm 0.003$ \\
$\rho_{\text {final }}$ & $-0.191 \pm 0.002$ & $-0.300 \pm 0.002$ & $-0.328 \pm 0.002$ & $-0.299 \pm 0.002$ & $-0.190 \pm 0.002$ \\
\hline \hline
\end{tabular}

heavier than nonstrange baryons, so their rapidity distributions are narrower. The kaon rapidity distribution is then compared to baryons, again wider. A change in baryon number (strangeness) at high $|y|$ is less likely to be accompanied by a change in strangeness (baryon number) than at low $|y|$. The value of $\rho_{B S}$, therefore, drops toward higher rapidity, as shown in Fig. 5 (bottom). By the same argument, we find a weakening of the baryon-electric charge correlation $\rho_{B Q}$ at higher rapidity [Fig. 6 (bottom)] as the rapidity distribution of electrically charged particles is wider than that of baryons. For the strangeness-electric charge correlation coefficient we find first a mild rise and then a somewhat stronger drop of $\rho_{S Q}$ toward higher rapidity. As one shifts ones acceptance window toward higher values of $|y|$, first the contribution of baryons (in particular $\Sigma^{+}$) decreases and, as the meson contribution grows, $\rho_{S Q}$ rises slightly. Toward the highest $|y|$, pions again dominate and decorrelate the quantum numbers.

The transverse momentum dependence can be understood as follows: heavier particles have higher average transverse momentum $\left\langle p_{T}\right\rangle$ and, hence, their influence increases toward higher $p_{T}$. Heavy particles have a tendency to carry several charges, causing the correlation coefficients to grow.

The contribution of strange baryons compared to nonstrange baryons grows toward higher transverse momentum, as strange baryons have on average larger mass than nonstrange baryons. The correlation coefficient $\rho_{B S}$ thus becomes strongly negative at high $p_{T}$. As the contribution of baryons compared to mesons grows stronger toward larger $p_{T}$, a change in baryon number (electric charge) is now more likely to be accompanied by a change in electric charge

TABLE III. Baryon-electric charge correlation coefficient $\rho_{B Q}$ in the GCE in transverse momentum bins $\Delta p_{T, i}$ and rapidity bins $\Delta y_{i}$, both primordial and final state.

\begin{tabular}{lccccc}
\hline \hline$\rho_{B Q}$ & $\Delta p_{T, 1}$ & $\Delta p_{T, 2}$ & $\Delta p_{T, 3}$ & $\Delta p_{T, 4}$ & $\Delta p_{T, 5}$ \\
\hline$\rho_{\text {prim }}^{\text {calc }}$ & 0.1120 & 0.1271 & 0.1420 & 0.1579 & 0.1781 \\
$\rho_{\text {prim }}$ & $0.113 \pm 0.002$ & $0.126 \pm 0.002$ & $0.143 \pm 0.003$ & $0.158 \pm 0.002$ & $0.178 \pm 0.003$ \\
$\rho_{\text {final }}$ & $0.112 \pm 0.003$ & $0.120 \pm 0.003$ & $0.138 \pm 0.003$ & $0.164 \pm 0.003$ & $0.221 \pm 0.003$ \\
$\rho_{B Q}$ & $\Delta y_{1}$ & $\Delta y_{2}$ & $\Delta y_{3}$ & $\Delta y_{4}$ & $\Delta y_{5}$ \\
$\rho_{\text {prim }}^{\text {calc }}$ & 0.1160 & 0.1601 & 0.1658 & 0.1601 & 0.1160 \\
$\rho_{\text {prim }}$ & $0.116 \pm 0.002$ & $0.160 \pm 0.003$ & $0.166 \pm 0.003$ & $0.159 \pm 0.003$ & $0.117 \pm 0.002$ \\
$\rho_{\text {final }}$ & $0.118 \pm 0.003$ & $0.192 \pm 0.003$ & $0.202 \pm 0.003$ & $0.192 \pm 0.003$ & $0.119 \pm 0.003$ \\
\hline \hline
\end{tabular}

TABLE IV. Strangeness-electric charge correlation coefficient $\rho_{S Q}$ in the GCE in transverse momentum bins $\Delta p_{T, i}$ and rapidity bins $\Delta y_{i}$, both primordial and final state.

\begin{tabular}{lccccc}
\hline \hline$\rho_{S Q}$ & $\Delta p_{T, 1}$ & $\Delta p_{T, 2}$ & $\Delta p_{T, 3}$ & $\Delta p_{T, 4}$ & $\Delta p_{T, 5}$ \\
\hline$\rho_{\text {prim }}^{\text {calc }}$ & 0.2831 & 0.3033 & 0.3150 & 0.3185 & 0.3055 \\
$\rho_{\text {prim }}$ & $0.284 \pm 0.003$ & $0.304 \pm 0.003$ & $0.314 \pm 0.003$ & $0.319 \pm 0.002$ & $0.305 \pm 0.002$ \\
$\rho_{\text {final }}$ & $0.243 \pm 0.003$ & $0.254 \pm 0.003$ & $0.276 \pm 0.003$ & $0.292 \pm 0.003$ & $0.303 \pm 0.002$ \\
$\rho_{S Q}$ & $\Delta y_{1}$ & $\Delta y_{2}$ & $\Delta y_{3}$ & $\Delta y_{4}$ & $\Delta y_{5}$ \\
$\rho_{\text {prim }}^{\text {calc }}$ & 0.2934 & 0.3137 & 0.3104 & 0.3137 & 0.2934 \\
$\rho_{\text {prim }}$ & $0.294 \pm 0.003$ & $0.314 \pm 0.003$ & $0.310 \pm 0.002$ & $0.312 \pm 0.003$ & $0.292 \pm 0.002$ \\
$\rho_{\text {final }}$ & $0.255 \pm 0.002$ & $0.299 \pm 0.003$ & $0.297 \pm 0.003$ & $0.298 \pm 0.003$ & $0.255 \pm 0.003$ \\
\hline \hline
\end{tabular}


(baryon number) than at low $p_{T}$, and $\rho_{B}$ increases with $p_{T}$ (The $\Delta$ resonances ${ }^{5}$ ensure it keeps rising). For the $\Delta p_{T, i}$ dependence of $\rho_{S O}$ we finally note that one of the strongest contributors at higher $p_{T}$ is the $\Omega^{-}$, with a relatively low mass of $m_{\Omega^{-}}=1.672 \mathrm{GeV}$. So after a rise, $\rho_{S Q}$ drops again toward highest $p_{T}$, due to an increasing $\Sigma^{+}$contribution. ${ }^{6}$

Because resonance decay has the habit of dropping the lighter particles (mesons) at low $p_{T}$ and higher $|y|$, while keeping heavier particles (baryons) at higher $p_{T}$ and at midrapidity, none of the above arguments about the transverse momentum and rapidity dependence are essentially changed by resonance decay. The correlation coefficient $\rho_{B S}$ becomes more negative toward higher $p_{T}$, while becoming weaker toward higher $|y|$. Similarly, $\rho_{B} Q$ grows larger at high $p_{T}$ and drops toward higher $y$. The larger contributions of baryons to the high $p_{T}$ tail of the transverse momentum spectrum, and their decreased contribution to the tails of the rapidity distribution, compared to mesons, are to blame. The bump in the $p_{T}$ dependence of $\rho_{S Q}$, presumably caused by the $\Sigma^{+}$, has vanished, as the $\Sigma^{+}$is only considered as stable in its lightest version with mass $m_{\Sigma^{+}}=1.189 \mathrm{GeV}$. The small bump in the $y$ dependence of $\rho_{S Q}$, however, stays. The correlation is presumably first increased by a growing kaon contribution and then again decreased by a growing pion contribution at larger rapidities.

The values of $\rho$ after resonance decay are directly sensitive to how the data are analyzed. In the above study we analyzed final state particles (stable against strong decays) only. One could, however, also reconstruct decay positions and momenta of parent resonances and could then count them as belonging to the acceptance bin the parent momentum would fall into. In the situation above, however, this would again yield the primordial scenario. If reconstruction of resonances is not done, one is sensitive to charge correlations carried by final state particles. As in the primordial case, a larger acceptance bin effectively averages over smaller bins. However, the smaller the acceptance bin, the more information is lost due to resonance decay. In full acceptance, final state and primordial correlation coefficients ought to be the same, because quantum numbers (and energy momentum) are conserved in the decays of resonances.

\section{B. Extrapolating to the MCE}

We next consider the extrapolation to the MCE limit of variances and covariances and, hence, correlation coefficients, of joint distributions of charges in limited acceptance. The primordial joint baryon number-strangeness distributions in different transverse momentum bins will serve as examples. In this subsection, we use an extended data set of $20 \times 8 \times 10^{5}$ events.

In Fig. 8 we show the evolution of the variances of the marginal primordial baryon number distribution $\left\langle(\Delta B)^{2}\right\rangle$ (top) and of the marginal primordial strangeness distribution $\left\langle(\Delta S)^{2}\right\rangle$ (bottom) in the transverse momentum bins $\Delta p_{T, i}$, defined in Table I, as a function of the size of the bath $\lambda=V_{1} / V_{g}$. Eight equally spaced values of $\lambda$ have been investigated. The

\footnotetext{
${ }^{5}$ Included in the THERMUS particle table up to the $\Delta(2420)$.

${ }^{6}$ Included in the THERMUS particle table up to the $\Sigma(2030)$.
}
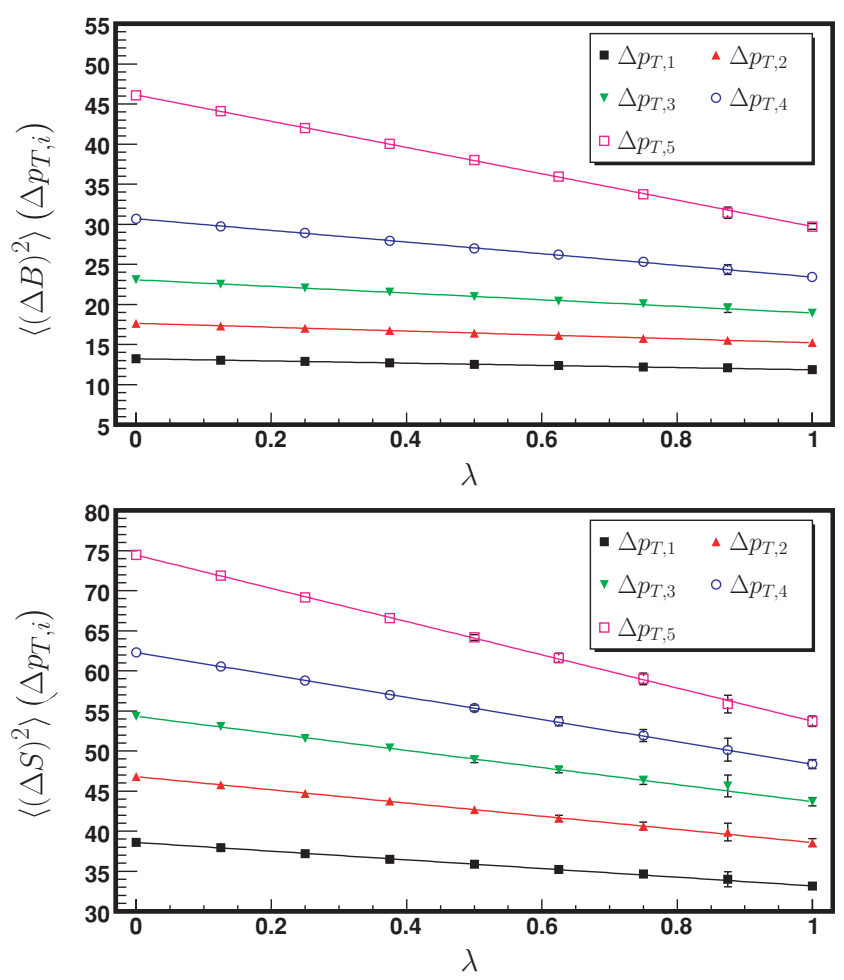

FIG. 8. (Color online) Evolution of the variance of the marginal baryon number distribution $\left\langle(\Delta B)^{2}\right\rangle$ (top) and the variance of the marginal strangeness distribution $\left\langle(\Delta S)^{2}\right\rangle$ (bottom) with $\lambda$ for a primordial hadron resonance gas in different $\Delta p_{T, i}$ bins. Each marker and its error bar except the last represents the result of 20 Monte Carlo runs of $10^{5}$ events each. Eight different equally spaced values of $\lambda$ have been investigated. The last marker denotes the result of the extrapolation. Solid lines indicate extrapolations from the GCE value to the MCE limit.

last marker denotes the result of the extrapolation. In Fig. 9 we show the dependence of the primordial covariance $\langle\Delta B \Delta S\rangle$ (top) and the primordial correlation coefficient $\rho_{B S}$ (bottom) of the joint baryon number-strangeness distribution on the size of the bath $\lambda$.

Let us first comment on the GCE values of variances (the left most markers in Fig. 8). As each of the five momentum bins holds one-fifth of the charged particle yield and, hence, less than one-fifth of the baryonic contribution in the lowest bin $\Delta p_{T, 1}$, and more than one-fifth in the highest bin $\Delta p_{T, 5}$, we find the baryon number variance $\left\langle(\Delta B)^{2}\right\rangle$ largest in $\Delta p_{T, 5}$ and smallest in $\Delta p_{T, 1}$. If binned in rapidity: $\Delta y_{3}$ has the strongest baryon contribution and, hence, $\left\langle(\Delta B)^{2}\right\rangle$ is largest there. The same goes for the variance $\left\langle(\Delta S)^{2}\right\rangle$ of the marginal strangeness distribution. Strangeness carrying particles are on average heavier than electrically charged particles and, hence, the strangeness contribution is strongest around midrapidity and toward larger transverse momentum (i.e., $\left\langle(\Delta S)^{2}\right\rangle$ is largest in $\Delta y_{3}$ and $\Delta p_{T, 5}$, while being smallest in $\Delta y_{1}, \Delta y_{5}$, and $\left.\Delta p_{T, 1}\right)$.

The $\Delta p_{T, i}$ dependence of the GCE covariance $\langle\Delta B \Delta S\rangle$ and the GCE correlation coefficient $\rho_{B S}$ in Fig. 9 is explained by the arguments of the previous subsection. Varying contributions of hadrons of different mass (and charge contents) to different parts of momentum space are responsible. 

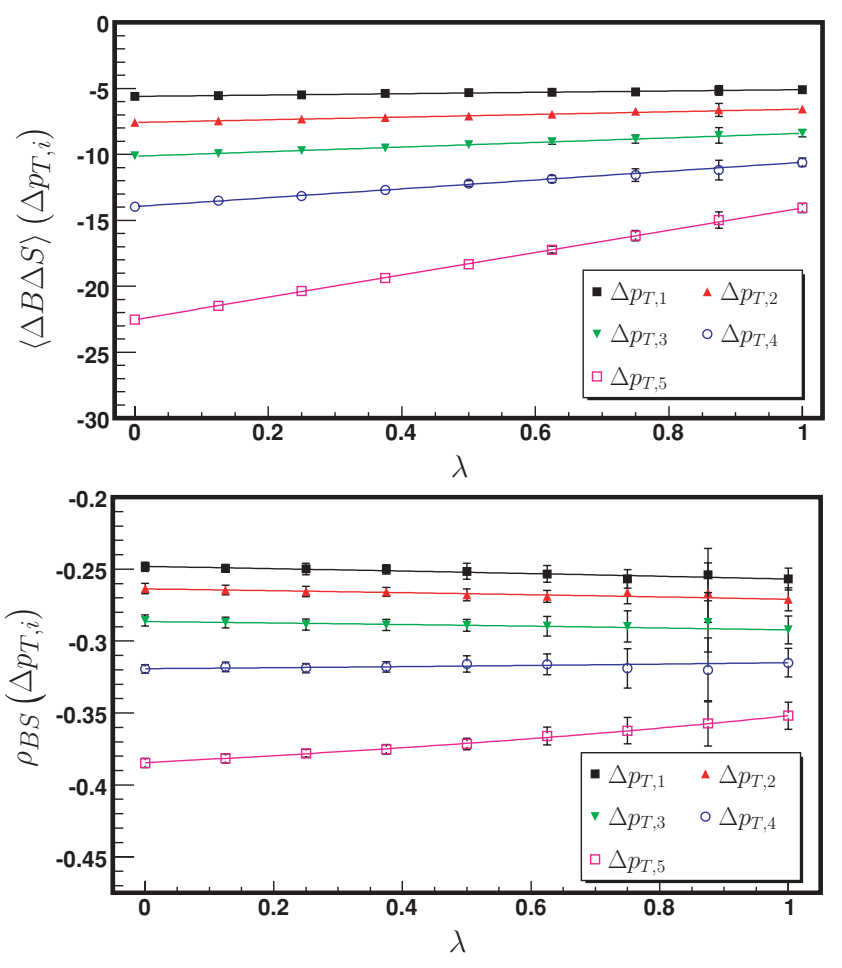

FIG. 9. (Color online) Evolution of the covariance $\langle\Delta B \Delta S\rangle$ (top) and the correlation coefficient $\rho_{B S}$ (bottom) of the baryon numberstrangeness distribution with $\lambda$ for a primordial hadron resonance gas in different $\Delta p_{T, i}$ bins; the rest as in Fig. 8.

We now turn our attention to the extrapolation. MCE effects on the baryonic sector are felt most strongly in momentumspace segments in which the baryonic contribution is strong (e.g., see the evolution of the last bin $\Delta p_{T, 5}$ with $\lambda$ in Figs. 8 and 9). The correlation coefficient is not as strongly affected, in general, by MCE effects.

In Fig. 10 we show the results of the extrapolation to the MCE limit of the baryon number-strangeness correlation coefficient $\rho_{B S}$ in acceptance bins $\Delta p_{T, i}$ and $\Delta y_{i}$, both primordial and final state. MCE values are closer to each other than corresponding GCE values, Fig. 5. The influence of globally applied conservation laws on charge correlations is less strong than for the multiplicity fluctuations and correlations discussed in the next section.

\section{MOMENTUM-SPACE DEPENDENCE OF MULTIPLICITY FLUCTUATIONS AND CORRELATIONS}

Multiplicity fluctuations and correlations are qualitatively affected by the choice of ensemble and are directly sensitive to the fraction of the system observed. For vanishing size of ones acceptance window, one would lose all information on how the multiplicities of any two distinct groups $N_{i}$ and $N_{j}$ of particles are correlated and measure $\rho_{i j}=0$. This information, however, is to some extent preserved in $\rho_{B S}, \rho_{B Q}$, and $\rho_{S Q}$, i.e., the way in which quantum numbers are correlated, if at least occasionally a particle is detected during an experiment.
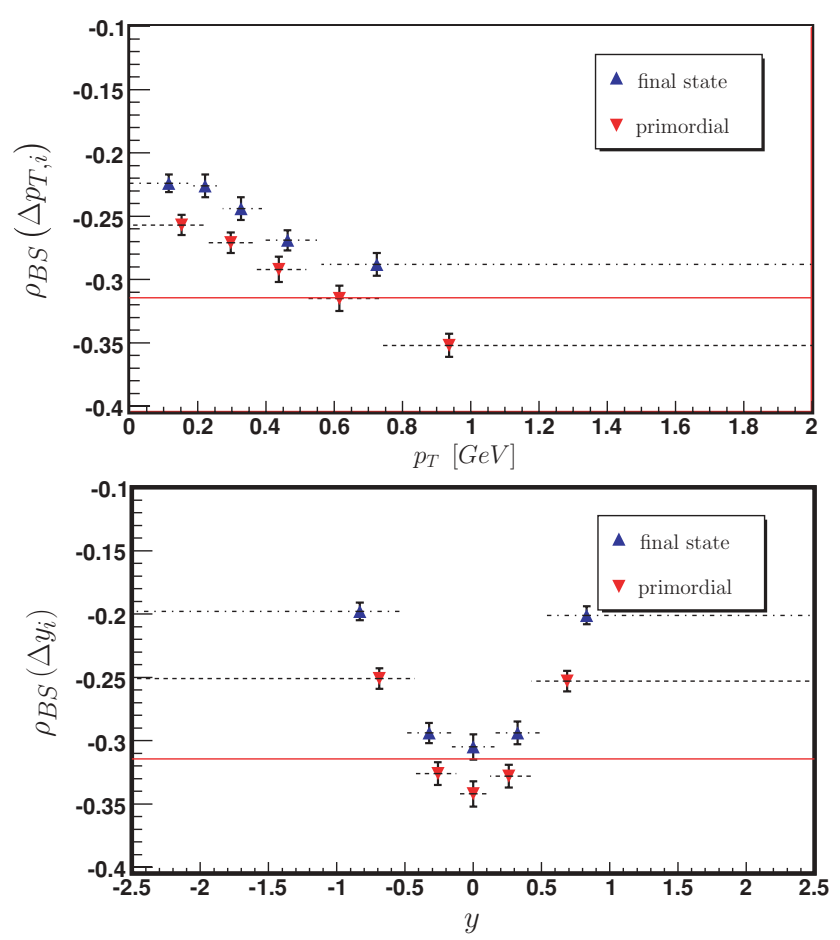

FIG. 10. (Color online) MCE baryon number-strangeness correlation coefficient $\rho_{B S}$ in limited acceptance windows, both primordial and final state. (Top) Transverse momentum bins $\Delta p_{T, i}$. (Bottom) Rapidity bins $\Delta y_{i}$. Horizontal error bars indicate the width and position of the momentum bins (and not an uncertainty!). Vertical error bars indicate the statistical uncertainty of the extrapolation of $8 \times 20$ Monte Carlo runs of $10^{5}$ events each. The marker indicates the center of gravity of the corresponding bin. The solid lines show the fully phase space integrated GCE result.

We first sample the same GCE system, which we have discussed in the previous sections, and consider the effects of resonance decay. Next the joint distributions of positively and negatively charged particles in momentum bins $\Delta p_{T, i}$ and $\Delta y_{i}$ are constructed. Then we, in turn, extrapolate the GCE primordial and final state results on the scaled variance $\omega$, Eq. (52), and the correlation coefficient $\rho$, Eq. (53), to the MCE limit.

\section{A. Grand canonical ensemble}

In Fig. 11 we show the $\Delta p_{T, i}$ (top) and $\Delta y_{i}$ (bottom) dependence of the GCE-scaled variance $\omega_{+}$of positively charged hadrons, both primordial and final state. In the primordial Boltzmann case one finds no dependence of multiplicity fluctuations on the position and size of the acceptance window. The observed multiplicity distribution is, within error bars, a Poissonian with scaled variance $\omega_{+}=1$. In fact, in the primordial GCE Boltzmann case any selection of particles has $\omega=1$.

In Fig. 12 we show the $\Delta p_{T, i}$ (top) and $\Delta y_{i}$ (bottom) dependence of the GCE correlation coefficient $\rho_{+-}$between positively and negatively charged hadrons, both primordial and final state. In the primordial Boltzmann case one finds also no dependence of multiplicity correlations on the position and 

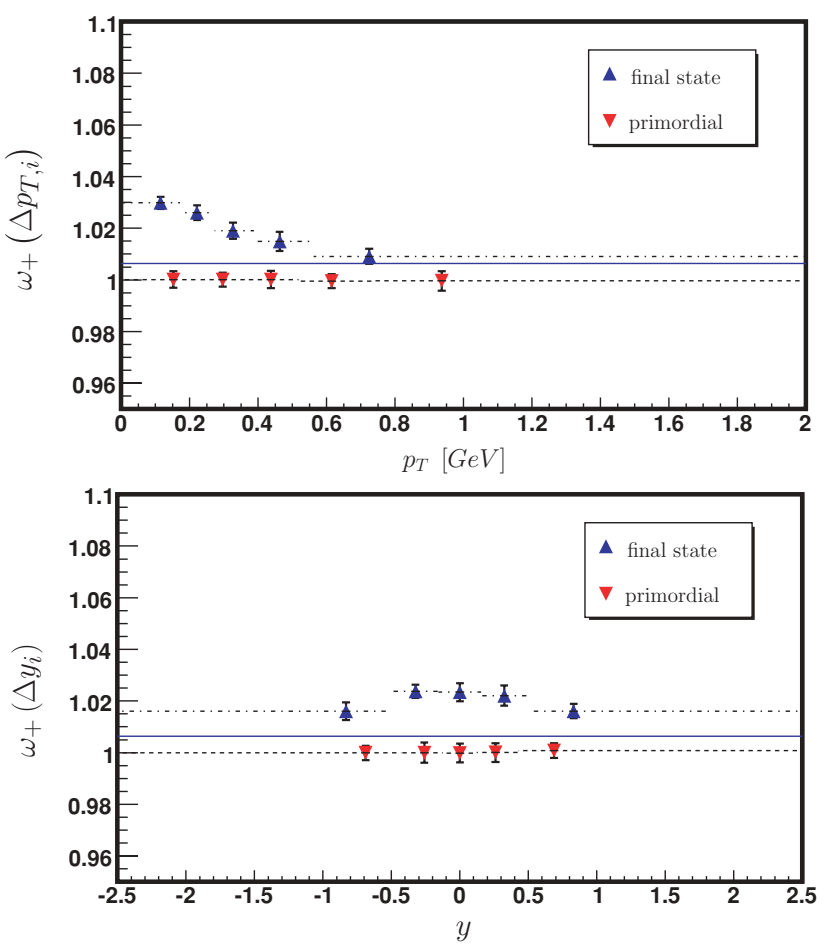

FIG. 11. (Color online) GCE-scaled variance $\omega_{+}$of multiplicity fluctuations of positively charged hadrons, both primordial and final state, in transverse momentum bins $\Delta p_{T, i}$ (top) and rapidity bins $\Delta y_{i}$ (bottom). Horizontal error bars indicate the width and position of the momentum bins (and not an uncertainty!). Vertical error bars indicate the statistical uncertainty of 20 Monte Carlo runs of $2 \times 10^{5}$ events each. The markers indicate the center of gravity of the corresponding bin. The solid line indicates the final state acceptance scaling estimate.

size of the acceptance window. The observed joint multiplicity distribution is a product of two Poissonians with correlation coefficient $\rho_{+-}=0$.

Resonance decay is the only source of correlation in an ideal GCE Boltzmann gas. Neutral hadrons decaying into two hadrons of opposite electric charge are the strongest contributors to the correlation coefficient $\rho_{+-}$. The chance that both (oppositely charged) decay products are dropped into the same momentum-space bin is obviously highest at low transverse momentum (i.e., the correlation coefficient is strongest in $\left.\Delta p_{T, 1}\right)$. The rapidity dependence is somewhat milder again, because heavier particles (parents) are dominantly produced at midrapidity and spread their daughter particles over a range in rapidity. One notes that the scaled variances and correlation coefficients in the respective acceptance bins in Figs. 11 and 12 are generally larger than the acceptance scaling procedure ${ }^{7}$ suggests, with the notable exception of $\rho_{+-}\left(\Delta p_{T, 5}\right)$.

If one would construct now a larger and larger number of momentum-space bins of equal average particle multiplicities,

\footnotetext{
${ }^{7}$ For the acceptance scaling approximation it is assumed that particles are randomly detected with a certain probability $q=0.2$, independent of their momentum.
}
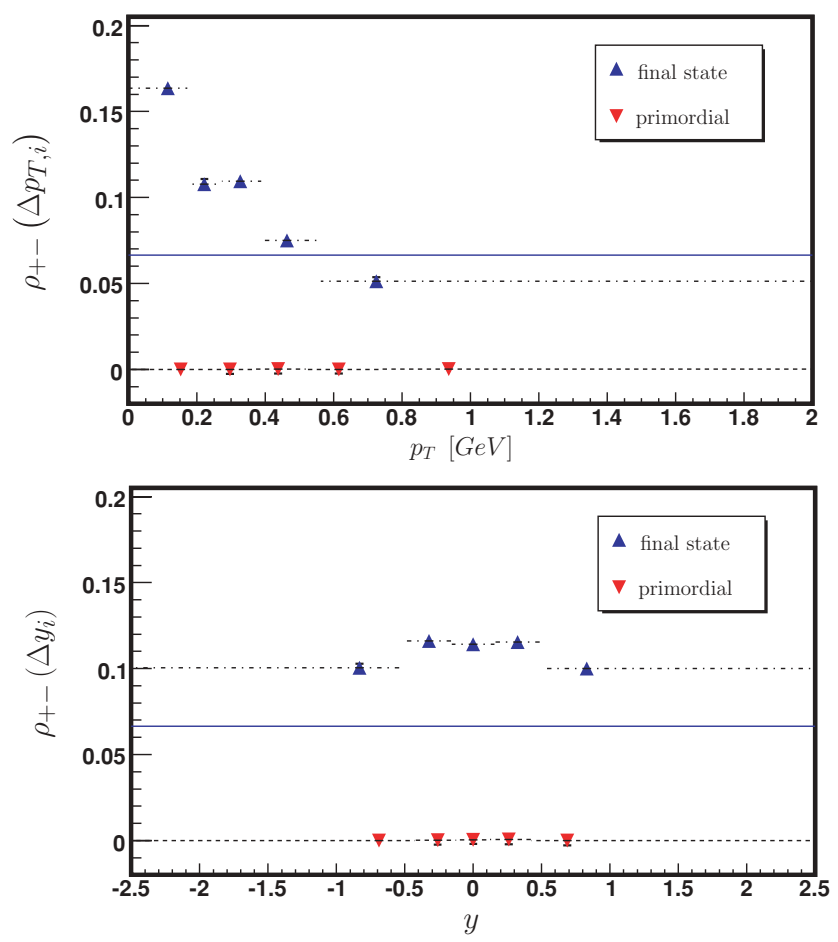

FIG. 12. (Color online) GCE multiplicity correlations $\rho_{+-}$between positively and negatively charged hadrons, both primordial and final state, in transverse momentum bins $\Delta p_{T, i}$ (top) and rapidity bins $\Delta y_{i}$ (bottom); the rest as in Fig. 11 .

one would successively lose more and more information about how multiplicities of distinct groups of particles are correlated.

There is a simple relation connecting the scaled variance of the fluctuations of all charged hadrons $\omega_{ \pm}$to the fluctuations of only positively charged particles $\omega_{+}$via the correlation coefficient $\rho_{+-}$between positively and negatively charged hadrons in a neutral system:

$$
\omega_{ \pm}=\omega_{+}\left(1+\rho_{+-}\right) .
$$

We, therefore, find the effect of resonance decay on the $\Delta p_{T, i}$ dependence of $\omega_{ \pm}$to be considerably stronger than on that of $\omega_{+}$, and generally $\omega_{ \pm}>\omega_{+}$, as the correlation coefficient $\rho_{+-}$ remains positive in the final state GCE. Compared to this, the final state values of $\omega_{ \pm}, \omega_{+}$, and $\rho_{+-}$remain rather flat with $\Delta y_{i}$ in the GCE.

\section{B. Extrapolating to the MCE}

In the very same way that we extrapolated fully phase space integrated extensive quantities to the MCE limit in Sec. IV, we now extrapolate multiplicity fluctuations $\omega_{+}$and correlations $\rho_{+-}$in transverse momentum bins $\Delta p_{T, i}$ and rapidity bins $\Delta y_{i}$ for a hadron resonance gas from the $\operatorname{GCE}(\lambda=0)$ to the MCE $(\lambda \rightarrow 1)$. Analytical primordial MCE results are done in the infinite volume approximation [21,22]. We, hence, have some guidance as to further asses the accuracy of the extrapolation scheme. For final state fluctuations and correlations in limited acceptance, however, no analytical results are available. 

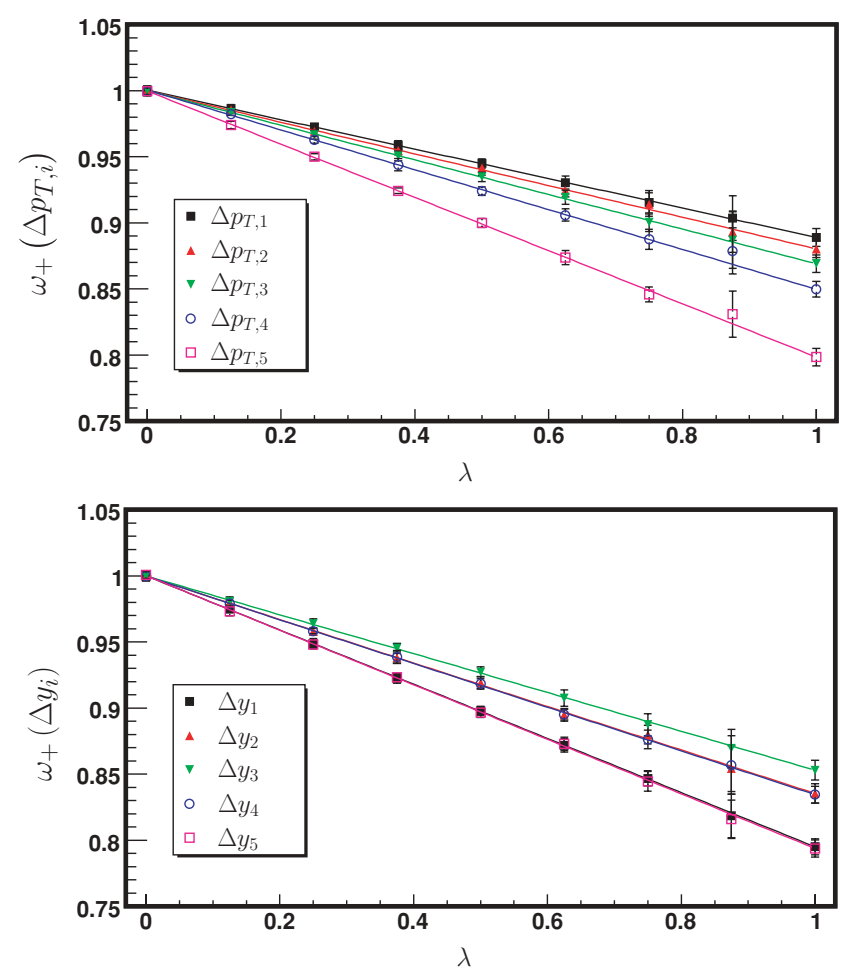

FIG. 13. (Color online) Evolution of the primordial scaled variance $\omega_{+}$of positively charged hadrons with the Monte Carlo parameter $\lambda=V_{1} / V_{g}$ for transverse momentum bins $\Delta p_{T, i}$ (top) and for rapidity bins $\Delta y_{i}$ (bottom). The solid lines show an analytic extrapolation from GCE results $(\lambda=0)$ to the MCE limit $(\lambda \rightarrow 1)$. Each marker and its error bar except the last represents the result of 20 Monte Carlo runs of $2 \times 10^{5}$ events. Eight different equally spaced values of $\lambda$ have been investigated. The last marker denotes the result of the extrapolation.

Mean values of particle numbers of positively charged hadrons $\left\langle N_{+}\right\rangle$and negatively charged hadrons $\left\langle N_{-}\right\rangle$in the respective acceptance bins, defined in Table I, remain constant as $\lambda$ goes from 0 to 1 , while the variances $\left\langle\left(\Delta N_{+}\right)^{2}\right\rangle$ and $\left\langle\left(\Delta N_{-}\right)^{2}\right\rangle$ and covariance $\left\langle\Delta N_{+} \Delta N_{-}\right\rangle$converge linearly to their respective MCE limits. The correlation coefficient $\rho_{+-}$ between positively and negatively charged hadrons, however, will not approach its MCE value linearly, as discussed in Sec. IV.

\section{Primordial}

In Fig. 13 we show the primordial scaled variance $\omega_{+}$ of positively charged hadrons in transverse momentum bins $\Delta p_{T, i}$ (top) and rapidity bins $\Delta y_{i}$ (bottom) as a function of the size of the bath $\lambda=V_{1} / V_{g}$, while in Fig. 14 we show the dependence of the primordial correlation coefficient $\rho_{+-}$between positively and negatively charged hadrons in transverse momentum bins $\Delta p_{T, i}$ (top) and rapidity bins $\Delta y_{i}$ (bottom) on $\lambda$.

The results of $8 \times 20$ Monte Carlo runs of $2 \times 10^{5}$ events each are summarized in Table $\mathrm{V}$. The system sampled was assumed to be neutral $\mu_{j}=(0,0,0)$ and static $u_{\mu}=$
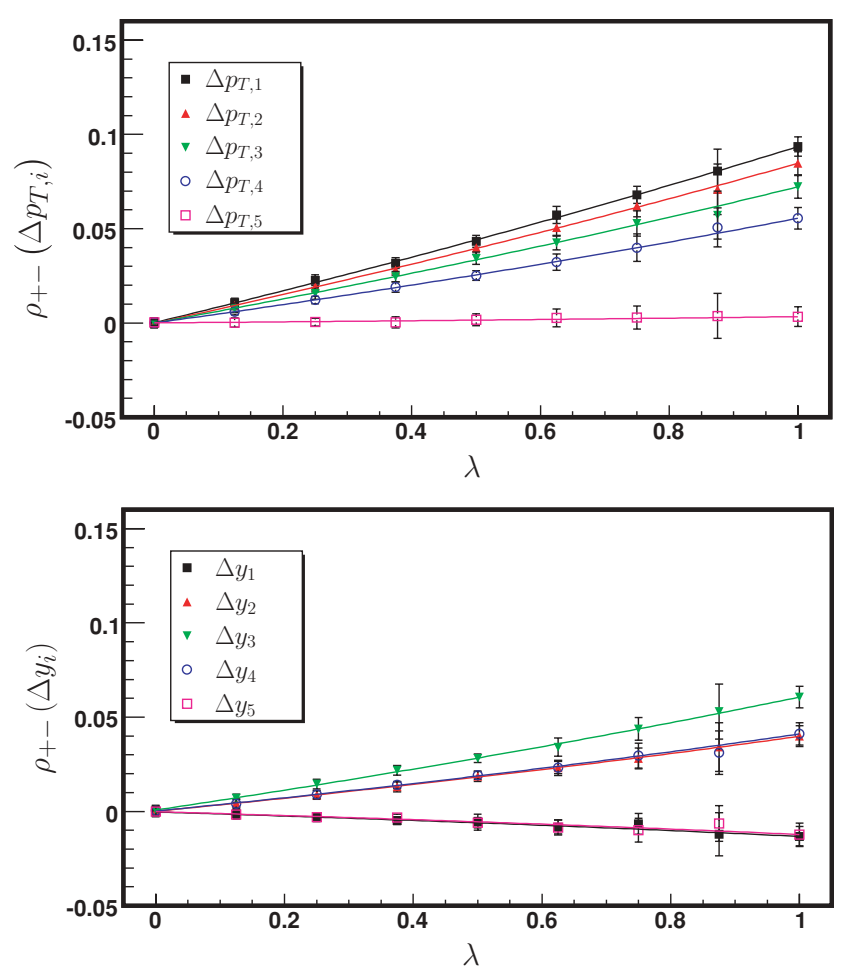

FIG. 14. (Color online) Evolution of the primordial correlation coefficient $\rho_{+-}$between positively and negatively charged hadrons with the Monte Carlo parameter $\lambda=V_{1} / V_{g}$ for transverse momentum bins $\Delta p_{T, i}$ (top) and for rapidity bins $\Delta y_{i}$ (bottom); the rest as in Fig. 13.

$(1,0,0,0)$ with local temperature $\beta^{-1}=0.160 \mathrm{GeV}$ and a system volume of $V_{1}=2000 \mathrm{fm}^{3}$. Eight different values of $\lambda$ have been studied. The last marker $(\lambda=1)$ denotes the result of the extrapolation. Only primordial hadrons are analyzed. Values for both $\Delta p_{T, i}$ and $\Delta y_{i}$ bins are listed. Analytical numbers are calculated according to the method developed in Refs. [21,22], using the acceptance bins defined in Table I, and are shown for comparison. The effects of energy-momentum and charge conservation on primordial multiplicity fluctuations and correlations in finite acceptance have been discussed in Refs. [21,22]. A few words attempt to summarize.

Let us first attend to fully phase space integrated results. The scaled variance of multiplicity fluctuations is lowest in the MCE due to the requirement of exact energy and charge conservation, somewhat larger in the $\mathrm{CE}$, and largest in the GCE, as now all constraints on the microstates of the system have been dropped $[27,30,31]$. The fully phase space integrated MCE and CE correlation coefficients between oppositely charged particles are rather close to 1 . Doubly charged particles allow for mild deviation, as also the $\Delta^{++}$resonance is counted as only one particle.

The transverse momentum dependence can be understood as follows: a change in particle number at high transverse momentum involves a large amount of energy, i.e., to balance the energy record, one needs to create (or annihilate) either a lighter particle with more kinetic energy or two particles at 
TABLE V. Summary of the primordial scaled variance $\omega_{+}$of positively charged hadrons and the correlation coefficient $\rho_{+-}$between positively and negatively charged hadrons in transverse momentum bins $\Delta p_{T, i}$ and rapidity bins $\Delta y_{i}$. Both the GCE result $(\lambda=0)$ and the extrapolation to $\operatorname{MCE}(\lambda=1)$ are shown. The uncertainty quoted corresponds to 20 Monte Carlo runs of $2 \times 10^{5}$ events (GCE) or is the result of the extrapolation (MCE). Analytic MCE results $\omega_{+}^{\text {mce, } c}$ and $\rho_{+-}^{\text {mce }, c}$ are listed too.

\begin{tabular}{lccccc}
\hline \hline Primordial & $\Delta p_{T, 1}$ & $\Delta p_{T, 2}$ & $\Delta p_{T, 3}$ & $\Delta p_{T, 4}$ & $\Delta p_{T, 5}$ \\
\hline$\omega_{+}^{\text {gce }}$ & $1.000 \pm 0.002$ & $1.000 \pm 0.002$ & $1.000 \pm 0.002$ & $1.000 \pm 0.002$ & $1.000 \pm 0.002$ \\
$\omega_{+}^{\text {mce }}$ & $0.889 \pm 0.007$ & $0.880 \pm 0.007$ & $0.869 \pm 0.007$ & $0.850 \pm 0.006$ & $0.798 \pm 0.007$ \\
$\omega_{+}^{\text {mce }, c}$ & 0.8886 & 0.8802 & 0.8682 & 0.8489 & 0.7980 \\
$\rho_{+-}^{\text {gce }}$ & $0.000 \pm 0.002$ & $-0.000 \pm 0.002$ & $-0.000 \pm 0.002$ & $0.000 \pm 0.002$ & $0.000 \pm 0.001$ \\
$\rho_{+-}^{\text {mce }}$ & $0.094 \pm 0.005$ & $0.085 \pm 0.006$ & $0.072 \pm 0.006$ & $0.056 \pm 0.006$ & $0.003 \pm 0.005$ \\
$\rho_{+-}^{\text {mce }, c}$ & 0.0935 & 0.0844 & 0.0730 & 0.0554 & 0.0040 \\
Primordial & $\Delta y_{1}$ & $\Delta y_{2}$ & $\Delta y_{3}$ & $\Delta y_{4}$ & $\Delta y_{5}$ \\
$\omega_{+}^{\text {gce }}$ & $1.000 \pm 0.002$ & $1.000 \pm 0.002$ & $1.000 \pm 0.003$ & $1.000 \pm 0.002$ & $1.000 \pm 0.002$ \\
$\omega_{+}^{\text {mce }}$ & $0.795 \pm 0.006$ & $0.835 \pm 0.007$ & $0.853 \pm 0.008$ & $0.834 \pm 0.006$ & $0.794 \pm 0.007$ \\
$\omega_{+}^{\text {mce, } c}$ & 0.7950 & 0.8350 & 0.8521 & 0.8351 & 0.7949 \\
$\rho_{+-}^{\text {gce }}$ & $-0.000 \pm 0.001$ & $0.000 \pm 0.002$ & $0.001 \pm 0.002$ & $0.000 \pm 0.002$ & $-0.000 \pm 0.002$ \\
$\rho_{+-}^{\text {mce }}$ & $-0.013 \pm 0.005$ & $0.040 \pm 0.006$ & $0.061 \pm 0.006$ & $0.041 \pm 0.006$ & $-0.012 \pm 0.006$ \\
$\rho_{+-}^{\text {mce }, c}$ & -0.0135 & 0.0406 & 0.0616 & 0.0406 & -0.0135 \\
\hline \hline
\end{tabular}

lower $p_{T}$. This leads to suppressed multiplicity fluctuations in high- $\Delta p_{T, i}$ bins compared to low- $\Delta p_{T, i}$ bins. By the same argument, it seems favorable, due to the constraint of energy and charge conservation, to balance electric charge, by creating (or annihilating) pairs of oppositely charged particles, predominantly in lower $\Delta p_{T, i}$ bins, while allowing for a more uncorrelated multiplicity distribution, i.e., also larger netcharge ( $\delta Q=N_{+}-N_{-}$) fluctuations, in higher- $\Delta p_{T, i}$ bins.

For the rapidity dependence similar arguments hold. Here, however, the strongest role is played by longitudinalmomentum conservation. A change in particle number at high $y$ involves now, in addition to a large amount of energy, a large momentum $p_{z}$ to be balanced. The constraints of global $P_{z}$ conservation are, hence, felt least severely around $|y| \sim 0$, and it becomes favorable to balance charge predominantly at midrapidity $\left(\rho_{+-}\right.$larger) and allow for stronger multiplicity fluctuations $\left(\omega_{+}\right.$larger) compared to forward and backward rapidity bins.

In a somewhat casual way one could say: events of a neutral hadron resonance gas with values of extensive quantities $B, S, Q, E$, and $P_{z}$ in the vicinity of $\left\langle\mathcal{Q}_{1}^{l}\right\rangle$ have a tendency to have similar numbers of positively and negatively charged particles at low transverse momentum $p_{T}$ and rapidity $y$ and less strongly so at high $p_{T}$ and $|y|$.

The statistical error on the "data" points grows as $\lambda \rightarrow 1$, as can be seen from Figs. 13 and 14. The extrapolation helps greatly to keep the statistical uncertainty on the MCE limit low, as summarized in Table $\mathrm{V}$, and can be seen from a comparison of the last two data points in Figs. 13 and 14. The last point and its error bar denote the result of a linear extrapolation of variances and covariances, while the second to last data point and its error bar are the result of 20 Monte Carlo runs with $\lambda=0.875$. The analytical MCE values are well within error bars of extrapolated Monte Carlo results and agree surprisingly well, given the large number of "conserved" quantities (5) and a relatively small sample size of $8 \times 20 \times 2 \times 10^{5}=3.2 \times$ $10^{7}$ events. In a sample-reject type of approach this sample size would yield a substantially larger statistical error, as only events with exact values of extensive quantities are kept for the analysis. As the system size is increased, a sample-reject formalism, hence, becomes increasingly inefficient, while the extrapolation method still yields good results. For a further discussion see Appendix A.

\section{Final state}

We now attend to the extrapolation of final state multiplicity fluctuations and correlations to the MCE limit. An independent Monte Carlo run for the same physical system was done but now with only stable final state particles "detected."

In Fig. 15 we show the final state scaled variance $\omega_{+}$ of positively charged hadrons in transverse momentum bins $\Delta p_{T, i}$ (top) and rapidity bins $\Delta y_{i}$ (bottom) as a function of $\lambda$, while in Fig. 16 we show the dependence of the final state correlation coefficient $\rho_{+-}$between positively and negatively charged hadrons in transverse momentum bins $\Delta p_{T, i}$ (top) and rapidity bins $\Delta y_{i}$ (bottom) on the size of the bath $\lambda=V_{1} / V_{g}$.

The $\Delta p_{T, i}$ and $\Delta y_{i}$ dependence on $\lambda$ of the final state MCE-scaled variance $\omega_{+}$is qualitatively similar to that of the primordial versions, Fig. 13, and is essentially also explained by the arguments of the previous section. The effects of charge and energy-momentum conservation work in pretty much the same way as before, and it still seems favorable to have events with wider multiplicity distributions at low $p_{T}$ and low $y$, and narrower distributions at larger $p_{T}$ and larger $|y|$. The dependence of the final state correlation coefficients $\rho_{+-}$on $\lambda$, 

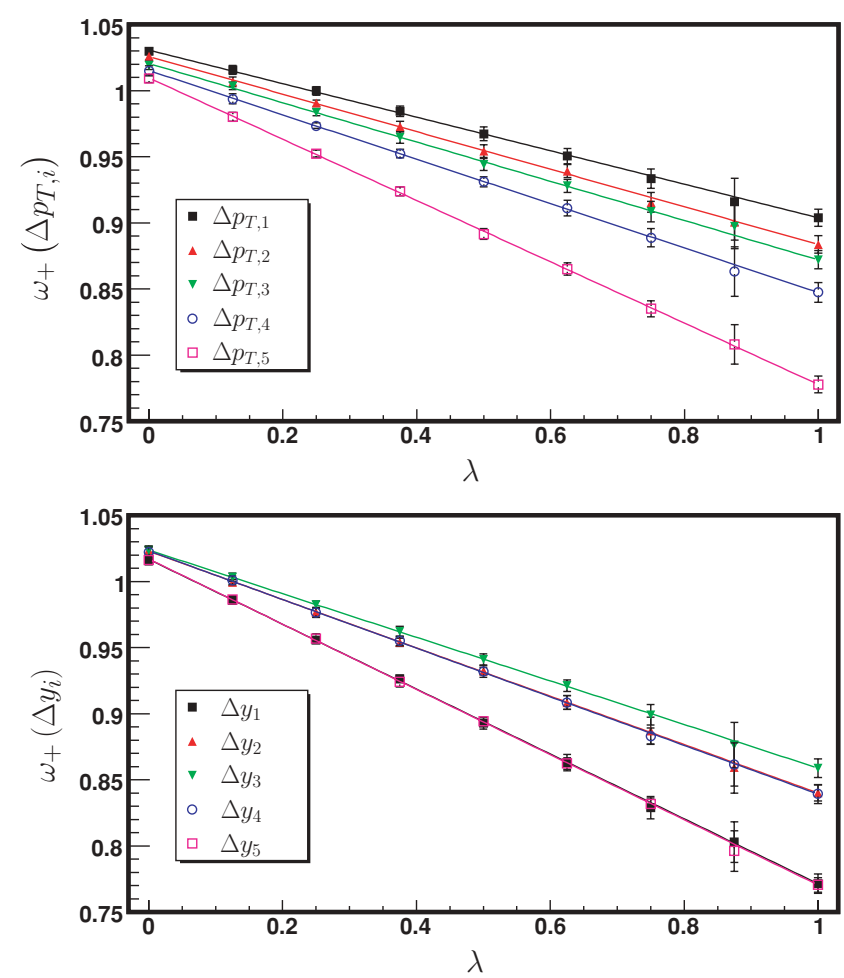

FIG. 15. (Color online) Evolution of the final state scaled variance $\omega_{+}$of positively charged hadrons with the Monte Carlo parameter $\lambda=V_{1} / V_{g}$ for transverse momentum bins $\Delta p_{T, i}$ (top) and for rapidity bins $\Delta y_{i}$ (bottom). The solid lines show an analytic extrapolation from GCE results $(\lambda=0)$ to the MCE limit $(\lambda \rightarrow 1)$. Each marker except the last represents the result of 20 Monte Carlo runs of $2 \times 10^{5}$ events. Eight different equally spaced values of $\lambda$ have been investigated. The last marker denotes the result of the extrapolation.

Fig. 16, is a bit different to the primordial case, Fig. 14. However, in the MCE limit, events still tend to have more similar numbers of oppositely charged particles at low $p_{T}$ and low $y$ than at large $p_{T}$ and large $|y|$.

The effects of resonance decay are qualitatively different in the MCE, CE, and GCE. Let us again first attend to fully phase space integrated multiplicity fluctuations discussed in Refs. [30,31]. The final state scaled variance increases in the GCE and CE compared to the primordial scaled variance. Multiplicity fluctuations of neutral mesons remain unconstrained by conservation laws. However, they often decay into oppositely charged particles, which increases multiplicity fluctuations of pions, for instance. In the MCE, due to the constraint of energy conservation, the event-byevent fluctuations of primordial pions are correlated to the event-by-event fluctuations of, in general, primordial parent particles, and $\omega^{\text {final }}<\omega^{\text {prim }}$ is possible in the MCE.

In Fig. 17 and Fig. 18 we compare the final state $\Delta p_{T, i}$ (top) and $\Delta y_{i}$ (bottom) dependence of the MCE-scaled variance $\omega_{+}$and the MCE correlation coefficient $\rho_{+-}$, respectively, to their primordial counterparts. The results of $8 \times 20$ Monte Carlo runs of $2 \times 10^{5}$ events each for a static and neutral hadron resonance gas with $T=0.160 \mathrm{GeV}$ are summarized in Table VI.
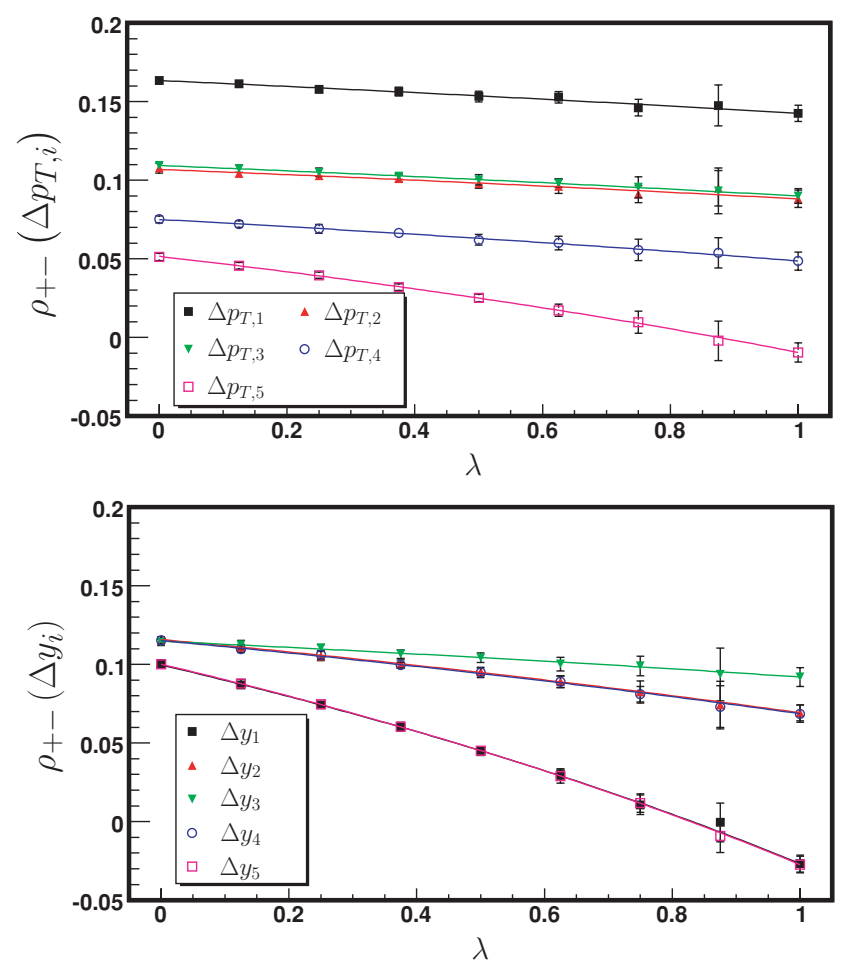

FIG. 16. (Color online) Evolution of the final state correlation coefficient $\rho_{+-}$between positively and negatively charged hadrons with the Monte Carlo parameter $\lambda=V_{1} / V_{g}$ for transverse momentum bins $\Delta p_{T, i}$ (top) and for rapidity bins $\Delta y_{i}$ (bottom); the rest as in Fig. 15.

A few words to summarize Figs. 17 and 18: resonance decay and (energy) conservation laws work in the same direction, as far as the transverse momentum dependence of the scaled variance $\omega_{+}$and the correlation coefficient $\rho_{+-}$is concerned. Both effects lead to increased multiplicity fluctuations and an increased correlation between the multiplicities of oppositely charged particles in the low $p_{T}$ region compared to the high $p_{T}$ domain.

Compared to this, the MCE $\Delta y_{i}$ dependence of $\omega_{+}$and $\rho_{+-}$ is mainly dominated by global conservation of $P_{z}$. Resonance decay effects, see Figs. 11 and 12, are more equal across rapidity than in transverse momentum.

Again, we find the scaled variance of all charged particles larger than the scaled variance of only positively charged hadrons $\omega_{ \pm}>\omega_{+}$, except for when $\rho_{+-}<0$, i.e., when the multiplicities of oppositely charged particles are anticorrelated, as for instance in $\Delta p_{T, 5}, \Delta y_{1}$, and $\Delta y_{5}$. In contrast to that, we narrowly find $\omega_{ \pm}>1$ in the lowest transverse momentum bin $\Delta p_{T, 1}$.

The qualitative picture presented in Fig. 17 could be compared to similar analysis of ultrarelativistic quantum molecular dynamics (UrQMD) transport simulation data [32], or recently published NA49 data on multiplicity fluctuations in limited momentum bins [33]. We, however, do not claim that the effects discussed above are the sole effects leading to the qualitative agreement with either of the two. 
TABLE VI. Summary of the final state scaled variance $\omega_{+}$of positively charged hadrons and the correlation coefficient $\rho_{+-}$ between positively and negatively charged hadrons in transverse momentum bins $\Delta p_{T, i}$ and rapidity bins $\Delta y_{i}$. Both the GCE result $(\lambda=0)$ and the extrapolation to the $\operatorname{MCE}(\lambda=1)$ are shown. The uncertainty quoted corresponds to 20 Monte Carlo runs of $2 \times 10^{5}$ events (GCE) or is the result of the extrapolation (MCE).

\begin{tabular}{lcccrr}
\hline \hline Final state & $\Delta p_{T, 1}$ & $\Delta p_{T, 2}$ & $\Delta p_{T, 3}$ & $\Delta p_{T, 4}$ & $\Delta p_{T, 5}$ \\
\hline$\omega_{+}^{\text {gce }}$ & $1.031 \pm 0.002$ & $1.026 \pm 0.002$ & $1.020 \pm 0.002$ & $1.015 \pm 0.002$ & $1.010 \pm 0.002$ \\
$\omega_{+}^{\text {mce }}$ & $0.904 \pm 0.007$ & $0.884 \pm 0.007$ & $0.872 \pm 0.007$ & $0.847 \pm 0.007$ & $0.778 \pm 0.006$ \\
$\rho_{+-}^{\text {gce }}$ & $0.163 \pm 0.001$ & $0.107 \pm 0.001$ & $0.109 \pm 0.001$ & $0.075 \pm 0.002$ & $0.052 \pm 0.002$ \\
$\rho_{+-}^{\text {mce }}$ & $0.143 \pm 0.005$ & $0.088 \pm 0.005$ & $0.090 \pm 0.005$ & $0.049 \pm 0.006$ & $-0.010 \pm 0.006$ \\
Final state & $\Delta y_{1}$ & $\Delta y_{2}$ & $\Delta y_{3}$ & $\Delta y_{4}$ & $\Delta y_{5}$ \\
$\omega_{+}^{\text {gce }}$ & $1.017 \pm 0.002$ & $1.023 \pm 0.002$ & $1.024 \pm 0.002$ & $1.023 \pm 0.003$ & $1.017 \pm 0.002$ \\
$\omega_{+}^{\text {mce }}$ & $0.771 \pm 0.007$ & $0.840 \pm 0.006$ & $0.859 \pm 0.007$ & $0.839 \pm 0.007$ & $0.770 \pm 0.006$ \\
$\rho_{+-}^{\text {gce }}$ & $0.100 \pm 0.001$ & $0.116 \pm 0.001$ & $0.115 \pm 0.002$ & $0.115 \pm 0.002$ & $0.100 \pm 0.001$ \\
$\rho_{+-}^{\text {mce }}$ & $-0.027 \pm 0.005$ & $0.069 \pm 0.005$ & $0.092 \pm 0.006$ & $0.069 \pm 0.006$ & $-0.027 \pm 0.005$ \\
\hline \hline
\end{tabular}

\section{SUMMARY}

We have presented a recipe for a thermal model Monte Carlo event generator capable of extrapolating fluctuation and correlation observables for Boltzmann systems of large volume from their GCE values to the MCE limit. Our approach has a strong advantage compared to analytical approaches
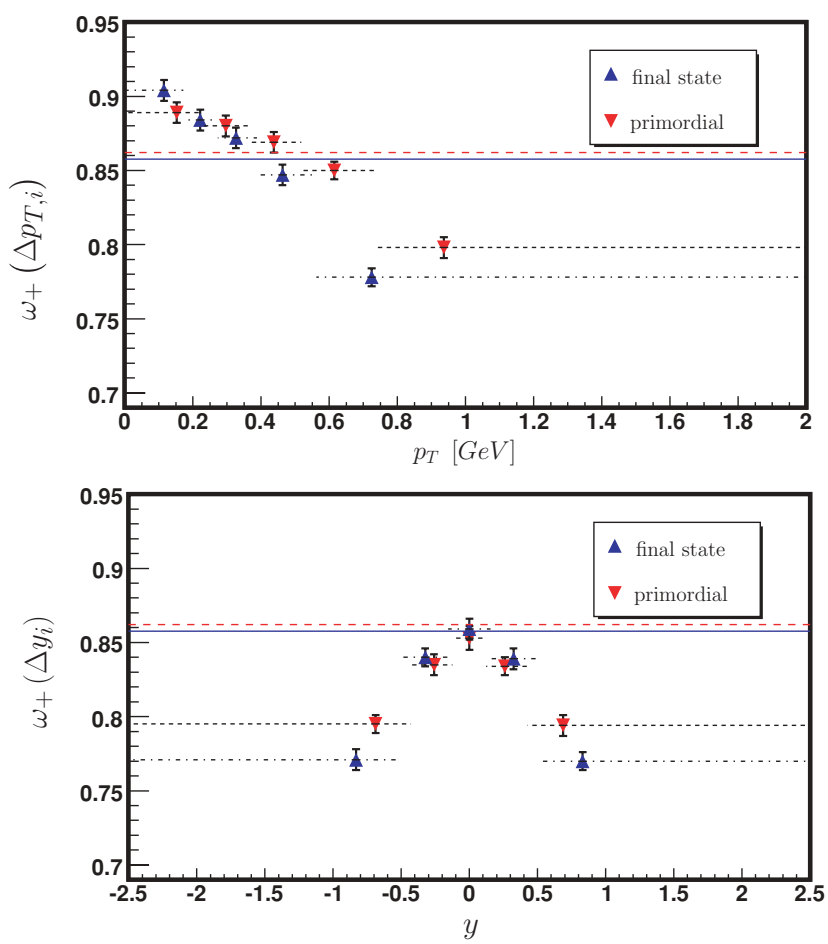

FIG. 17. (Color online) MCE-scaled variance $\omega_{+}$of multiplicity fluctuations of positively charged hadrons, both primordial and final state, in transverse momentum bins $\Delta p_{T, i}$ (top) and rapidity bins $\Delta y_{i}$ (bottom). Horizontal error bars indicate the width and position of the momentum bins (and not an uncertainty!). Vertical error bars indicate the statistical uncertainty quoted in Table VI. The markers indicate the center of gravity of the corresponding bin. The solid and the dashed lines show final state and primordial acceptance scaling estimates, respectively. or standard microcanonical sample-and-reject Monte Carlo techniques, in that it can handle resonance decays as well as (very) large system sizes at the same time.

To introduce our scheme, we have conceptually divided a microcanonical system into two subsystems. These subsystems are assumed to be in equilibrium with each other and subject to the constraints of joint energy-momentum and charge conservation. Particles are only measured in one subsystem, while the second subsystem provides a thermodynamic bath. By keeping the size of the first subsystem fixed, while varying the size of the second, one can thus study the dependence
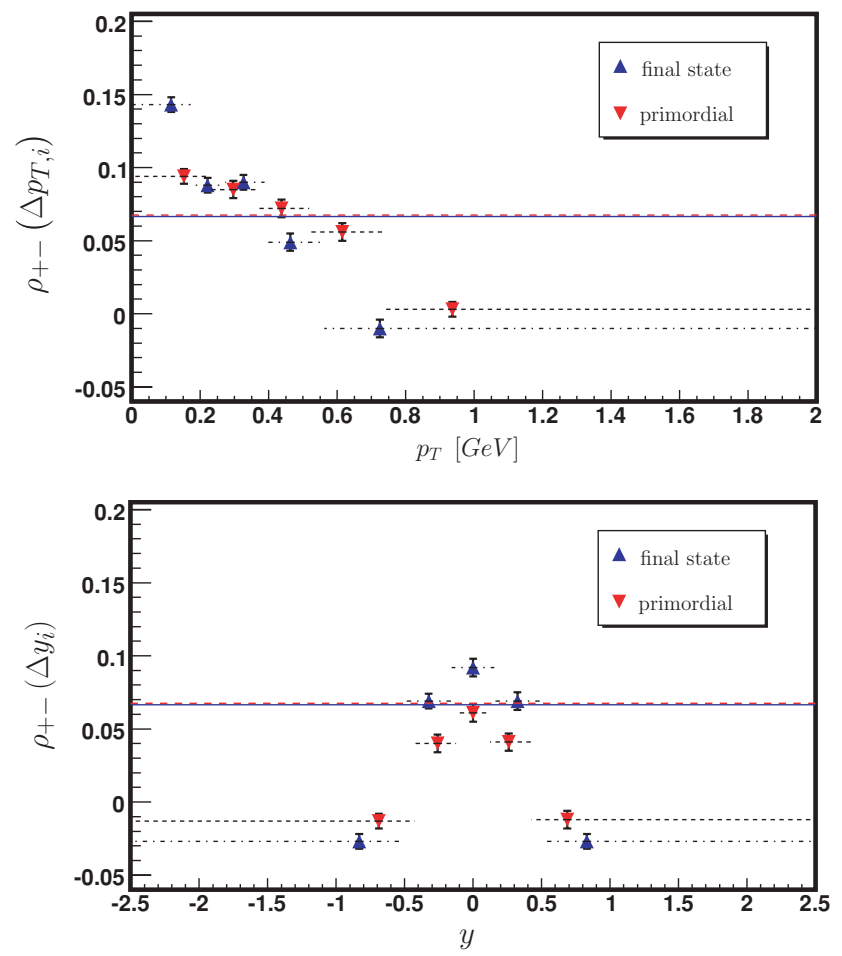

FIG. 18. (Color online) MCE multiplicity correlation coefficient $\rho_{+-}$between positively and negatively charged hadrons, both primordial and final state, in transverse momentum bins $\Delta p_{T, i}$ (top) and rapidity bins $\Delta y_{i}$ (bottom). The rest as in Fig. 17 . 
of statistical properties of an ensemble on the fraction of the system observed (i.e., assess their sensitivity to globally applied conservation laws). The ensembles generated are thermodynamically equivalent in the sense that mean values in the observed subsystem remain unchanged when the size of the bath is varied, provided the combined system is sufficiently large.

The Monte Carlo process can be divided into four steps. In the first two steps primordial particle multiplicities for each species, and momenta for each particle, are generated for each event by sampling the grand canonical partition function. In the third step resonance decay of unstable particles is performed. Last, the values of extensive quantities are calculated for each event and corresponding weight factor is assigned. All events with the same set of extensive quantities hence still have "a priori equal probabilities." In the limit of an infinite bath, all events have a weight equal to unity. In the opposite limit of a vanishing bath, only events with an exactly specified set of extensive quantities have nonvanishing weight. In between, we extrapolate in a controlled manner. The method is even rather efficient for large volume, inaccessible to sample-and-reject procedures, and agrees well, where available, with analytic asymptotic microcanonical solutions.

Given the success of the hadron resonance gas model in describing experimentally measured average hadron yields, and its ability to reproduce low temperature lattice susceptibilities, the question arises as to whether fluctuation and correlation observables also follow its main line. In particular, three effects are nicely discussable: resonance decay, conservation laws, and limited acceptance effects. Due to the Monte Carlo nature, data can be analyzed in close relation to experimental analysis techniques. The hadron resonance gas is an ideal testbed for this type of study in that it is simple and intuitive.

The statistical properties of a sample of hadron resonance gas events show a systematic dependence on what part of the momentum distribution and what fraction of the system is observed. Two examples served to illustrate: grand canonical charge-charge correlations and microcanonical multiplicity fluctuations and correlations. In the case of charge-charge correlations, momentum-space effects are caused by different masses of hadrons and, hence, their varying contribution to different parts of the momentum spectra. Although microcanonical effects on the (co-)variances of the joint baryon number-strangeness-electric charge distribution are considerable, they remain weak for the correlation coefficients between these quantum numbers. In contrast to this, momentum-space effects on multiplicity fluctuations and correlations arise due to conservations laws. For an ideal primordial grand canonical ensemble in the Boltzmann approximation (our starting point), multiplicity distributions are just uncorrelated Poissonians, regardless of the acceptance cuts applied, as particles are assumed to be produced independently. The requirement of energy-momentum and charge conservation leads to suppressed fluctuations and enhanced correlations between the multiplicities of two distinct groups of particles at the "high-momentum" end of the momentum spectrum, provided some fraction of an isolated system is observed. Resonance decay does not change these trends. The arguments on which the explanation of this particular dependence are based seem general enough to hope that they might hold too in nonequilibrium systems, such as real heavy-ion data or theoretical transport simulations.

A direct comparison with experimental data seems problematic at the moment. The static global thermal and chemical equilibrium assumption made here is certainly insufficient. The model presented here is far from complete. Several interesting aspects deserve attention. They include the sampling of Fermi-Dirac or Bose-Einstein particles, for which low transverse momentum is particularly sensitive; finite volume corrections could be done (possible if one has a good approximation to $\mathcal{W}$ ); the convergence properties (at fixed $\lambda$, and as a function of $\lambda$ ) fall basically into the same direction; so far we also have not derived a thermodynamic potential for our ensembles; one could also consider more general forms of $\mathcal{W}$; one could ask how to couple two systems of different densities, or altogether depart from the local equilibrium assumption. There are also several interesting things that the model could do in its present form. Examples include mean transverse momentum fluctuations, correlation between transverse momentum and particle number, or even two- and three-particle correlation functions. This should be the subject of future work.

\section{ACKNOWLEDGMENTS}

We thank F. Becattini, E. Bratkovskaya, W. Cassing, J. Cleymans, M. Gazdzicki, M. Gorenstein, J. Manninen, J. Randrup, and K. Redlich for fruitful discussions. Special thanks goes to W. Broniowski for his contribution to the very idea that started this project. The computational work was done on the CARMEN cluster of the UCT physics department. We also thank G. de Vaux for valuable help with many aspects of running the code.

\section{APPENDIX A: CONVERGENCE STUDY}

Not only for the sake of completeness we discuss in this section the convergence of various quantities with the sample size, i.e., the number of events, $N_{\text {events }}$, in our Monte Carlo scheme. Here we analyze final state (stable against electromagnetic and weak decays) particles only. We mainly take a closer look at the data subset of $20 \times 2 \times 10^{5}$ events, with $\lambda=V_{1} / V_{g}=0.875$ for the size of the bath, which already has been discussed in Sec. VII.

There is a degree of freedom of how to estimate the statistical uncertainty on the moments of a distribution of observables of a finite sample. The approach taken here is straightforward but could, however, certainly be improved.

In Fig. 19 we show the evolution of the mean values $\left\langle N_{+}\right\rangle$ (top) and the variances $\left\langle\left(\Delta N_{+}\right)^{2}\right\rangle$ (bottom) of the distributions of positively charged hadrons for the five transverse momentum bins $\Delta p_{T, i}$, defined in Table I, with the sample size. Mean values of particle multiplicities in respective bins are in rather good approximation equal to each other but are, however, not identical due to finite resolution on the underlying momentum spectrum, even for $\lambda=0.875$ (bins were constructed using GCE events from an independent run). Variances converge 

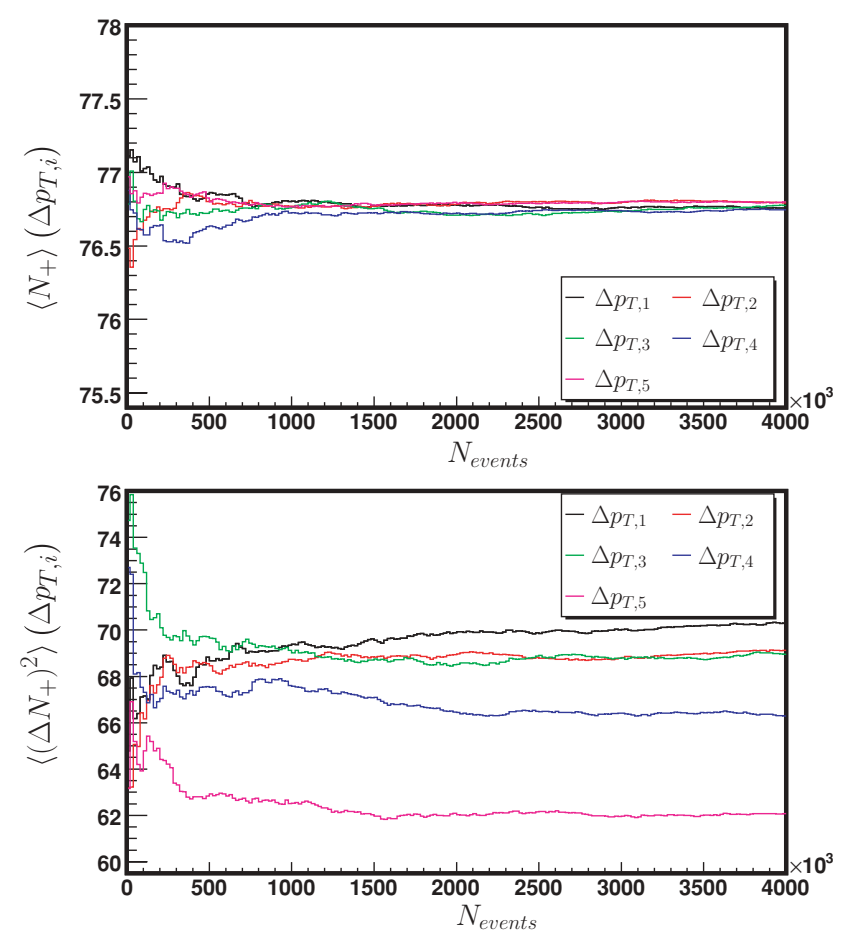

FIG. 19. (Color online) Step histogram showing the convergence of the mean values $\left\langle N_{+}\right\rangle$(top) and variances $\left\langle\left(\Delta N_{+}\right)^{2}\right\rangle$ (bottom) for positively charged final state hadrons in transverse momentum bins $\Delta p_{T, i}$ for a hadron resonance gas with $\lambda=V_{1} / V_{g}=0.875$.

steadily and are different in different bins, see Sec. VII. The event output was iteratively stored in histograms, which were then evaluated after steps of $2 \times 10^{4}$ events.

In Fig. 20 we show the evolution of the scaled variance $\omega_{+}$of positively charged final state particles (top) and the correlation coefficient $\rho_{+-}$between positively and negatively charged particles (bottom). The results for the respective transverse momentum bins can be compared to the secondto-last markers, Figs. 15 and 16 (top panels), which denote the corresponding results of grouping the same data into 20 Monte Carlo sets of $2 \times 10^{5}$ events each.

In Fig. 21 we show the distribution of scaled variances of positively charged particles $\omega_{+}$(top) and correlation coefficients between positively and negatively charged particles $\rho_{+-}$ (bottom), resulting from grouping again the same data set into 200 samples of $2 \times 10^{4}$ events each. We chose the transverse momentum bin $\Delta p_{T, 5}$ for a final state hadron resonance gas with $\lambda=V_{1} / V_{g}=0.875$.

Monte Carlo results for $\lambda=0.875$ of the analysis shown in Fig. 21, are for the scaled variance $\omega_{+}\left(\Delta p_{T, 5}\right)=0.8069 \pm$ 0.0514 , and the correlation coefficient $\rho_{+-}\left(\Delta p_{T, 5}\right)=$ $-0.0026 \pm 0.0421$. They are nicely scattered around the mean values, denoted by the bottom lines in Fig. $20, \omega_{+}\left(\Delta p_{T, 5}\right)=$ 0.8082 , and $\rho_{+-}\left(\Delta p_{T, 5}\right)=-0.0028$, respectively.

They are also compatible with the analysis shown in Figs. 15 and 16, of Sec. VII, $\omega_{+}\left(\Delta p_{T, 5}\right)=0.8081 \pm 0.0149$, and $\rho_{+-}\left(\Delta p_{T, 5}\right)=-0.0022 \pm 0.0125$, at the same value of $\lambda$. The comparatively large statistical error on the analysis in Fig. 21 is due to the splitting up into many small subsamples. The mean values of different analyses agree rather well.
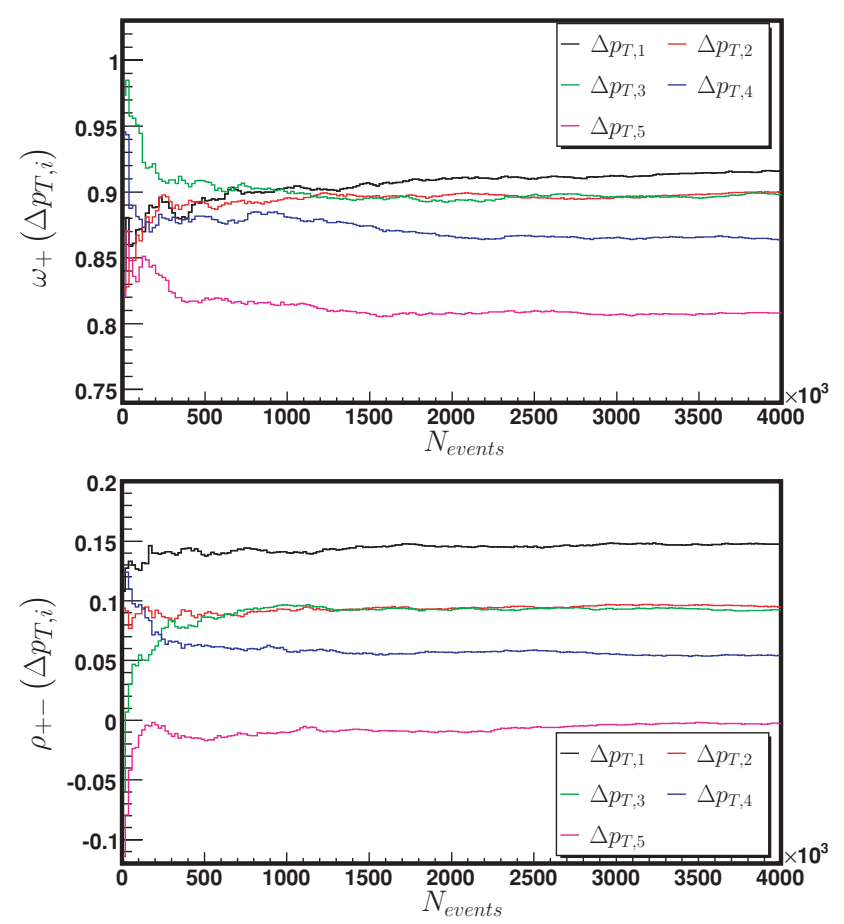

FIG. 20. (Color online) Step histogram showing the convergence of the scaled variance $\omega_{+}$(top) of positively charged hadrons and the correlation coefficient $\rho_{+-}$between positively and negatively charged hadrons (bottom) in transverse momentum bins $\Delta p_{T, i}$ for a final state hadron resonance gas with $\lambda=V_{1} / V_{g}=0.875$.

Last, we show in Fig. 22 the results of additional Monte Carlo runs for values of $\lambda$ closer to unity. This time we have performed 20 runs of $1 \times 10^{7}$ primordial events for $\lambda=0.925,0.950$, and 0.975. As discussed above, error bars diverge, but convergence seems to be rather good. The additional data has not been used for the extrapolation, so it can serve as an unbiased cross-check.

\section{APPENDIX B: THE CANONICAL BOLTZMANN GAS}

An analytical and instructive example is the canonical classical relativistic particle antiparticle gas discussed in Refs. $[29,37,38]$. We use this example to show that, although the procedure is formally independent of one's choice of Lagrange multipliers, it is most efficient for those defined by Maxwell's relations. We start off with Eq. (1) and then discuss, in turn, the first and second moments of the multiplicity distribution of particles and the first four moments of the Monte Carlo weight factor.

The canonical partition function $Z_{N_{1}}\left(V_{1}, \beta, Q_{1}\right)$ of a system with volume $V_{1}$, temperature $T=\beta^{-1}$, charge $Q_{1}$, particle number $N_{1}$, and antiparticle number $M_{1}=N_{1}-Q_{1}$, is given by

$$
Z_{N_{1}}\left(V_{1}, \beta, Q_{1}\right)=\frac{\left(V_{1} \psi\right)^{N_{1}}}{N_{1} !} \frac{\left(V_{1} \psi\right)^{N_{1}-Q_{1}}}{\left(N_{1}-Q_{1}\right) !} .
$$

The single-particle partition function is given by Eq. (31), $\psi=\frac{g}{2 \pi^{2}} m^{2} \beta^{-1} K_{2}(m \beta)$. The canonical partition function with arbitrary particle number, but still fixed charge $Q_{1}$, is obtained 

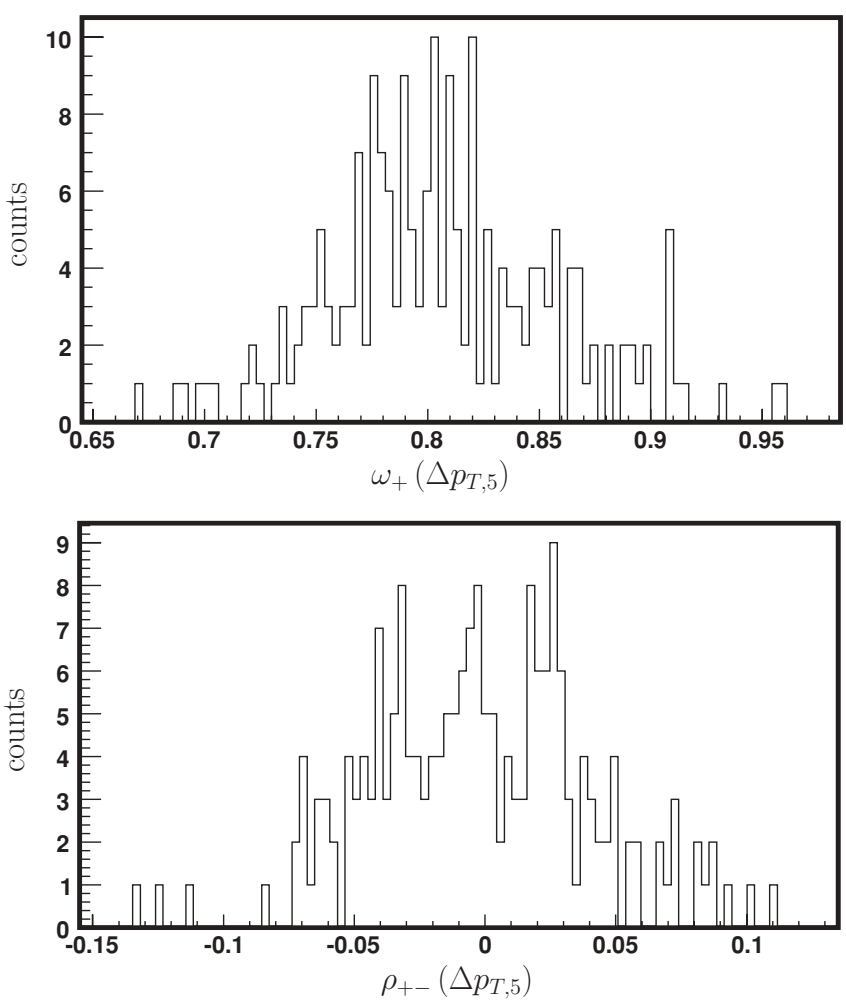

FIG. 21. Histogram showing the results for the scaled variance $\omega_{+}$ (top) of positively charged hadrons and the correlation coefficient $\rho_{+-}$ between positively and negatively charged hadrons (bottom) in the transverse momentum bin $\Delta p_{T, 5}$ for a final state hadron resonance gas with $\lambda=V_{1} / V_{g}=0.875$. Two hundred Monte Carlo runs of $2 \times 10^{4}$ events each are analyzed.

by:

$$
Z\left(V_{1}, \beta, Q_{1}\right)=\sum_{N_{1}=Q_{1}}^{\infty} Z_{N_{1}}\left(V_{1}, \beta, Q_{1}\right)=I_{Q_{1}}\left(2 V_{1} \psi\right) .
$$

Here $I_{Q_{1}}$ is a modified Bessel function. Temperature is the same in both subsystems; the bath and the observable part. The partition function of the bath is therefore

$$
Z\left(V_{2}, \beta, Q_{2}\right)=I_{Q_{2}}\left(2 V_{2} \psi\right) \text {. }
$$

Imposing the constraints $V_{2}=V_{g}-V_{1}$, and $Q_{2}=Q_{g}-Q_{1}$, similar to Eq. (4), we find [39] for the canonical partition function, Eq. (5), of the combined system:

$$
\begin{aligned}
Z\left(V_{g}, \beta, Q_{g}\right) & =\sum_{Q_{1}=-\infty}^{\infty} I_{Q_{1}}\left(2 V_{1} \psi\right) I_{Q_{g}-Q_{1}}\left[2\left(V_{g}-V_{1}\right) \psi\right] \\
& =I_{Q_{g}}\left(2 V_{g} \psi\right),
\end{aligned}
$$

as required. The weight factor is then:

$$
W\left(V_{1}, Q_{1} ; V_{g}, Q_{g} \mid \beta\right)=\frac{I_{Q_{g}-Q_{1}}\left[2\left(V_{g}-V_{1}\right) \psi\right]}{I_{Q_{g}}\left(2 V_{g} \psi\right)} .
$$

Analogous to Eq. (7) we find for the joint particle multiplicity and charge distribution:

$$
P\left(Q_{1}, N_{1}\right)=W\left(V_{1}, Q_{1} ; V_{g}, Q_{g} \mid \beta\right) Z_{N_{1}}\left(V_{1}, \beta, Q_{1}\right) .
$$
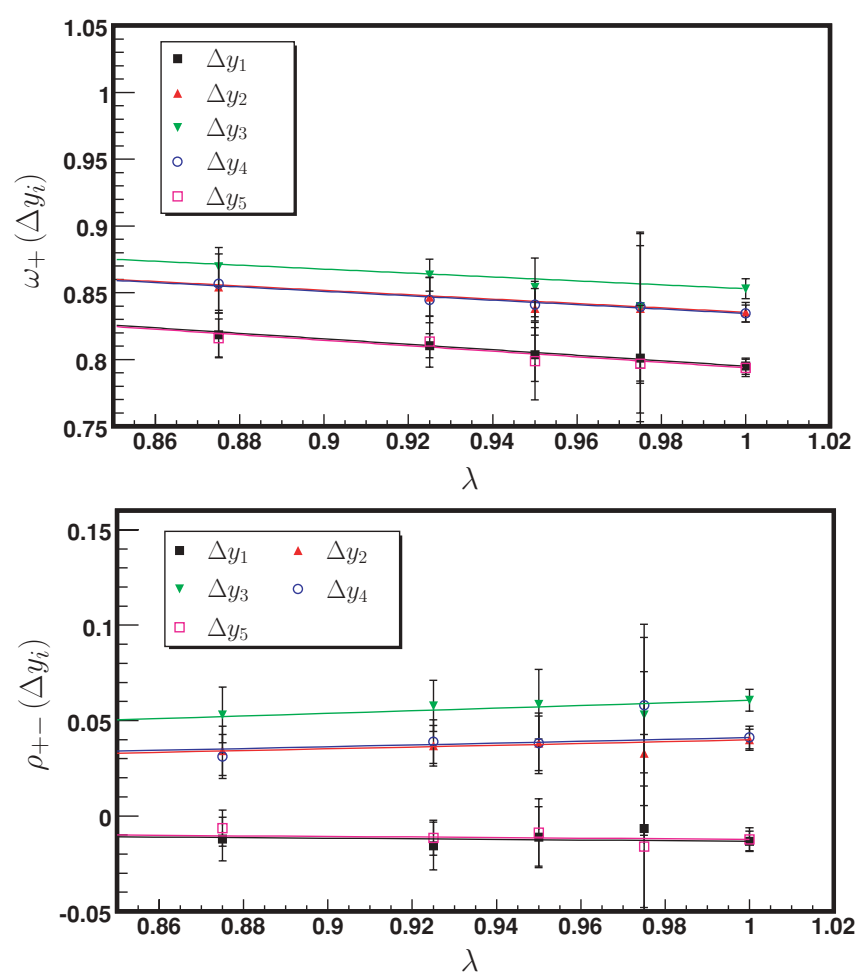

FIG. 22. (Color online) Evolution of the primordial scaled variance $\omega_{+}$of positively charged hadrons (top) and the primordial correlation coefficient $\rho_{+-}$between positively and negatively charged hadrons (bottom) with the Monte Carlo parameter $\lambda=V_{1} / V_{g}$ in different rapidity bins $\Delta y_{i}$. The solid lines show an analytic extrapolation from GCE results $(\lambda=0)$ to the MCE limit $(\lambda \rightarrow 1)$. The four leftmost markers and their error bars represent the results of 20 Monte Carlo runs of $2 \times 10^{5}$ events. Three additional values of $\lambda$ have been investigated with 20 Monte Carlo runs of $1 \times 10^{7}$ events. The rightmost markers denote the results of the extrapolation.

\section{Monte Carlo weight}

We next introduce Eq. (12), the joint GCE distribution of charges and particle multiplicity:

$$
P_{\text {gce }}\left(Q_{1}, N_{1}\right)=\frac{e^{Q_{1} \mu \beta}}{Z\left(V_{1}, \beta, \mu\right)} Z_{N_{1}}\left(V_{1}, \beta, Q_{1}\right) .
$$

The Monte Carlo weight, Eq. (15), is then given by

$$
\begin{aligned}
\mathcal{W}^{Q_{1} ; Q_{g}}\left(V_{1} ; V_{g} \mid \beta, \mu\right) \equiv & W\left(V_{1}, Q_{1} ; V_{g}, Q_{g} \mid \beta\right) \\
& \times Z\left(V_{1}, \beta, \mu\right) e^{-Q_{1} \mu \beta} .
\end{aligned}
$$

In accordance with Eq. (11), the distribution Eq. (B6) is then equivalently written as

$$
P\left(Q_{1}, N_{1}\right)=\mathcal{W}^{Q_{1} ; Q_{g}}\left(V_{1} ; V_{g} \mid \mu, \beta\right) P_{\text {gce }}\left(Q_{1}, N_{1}\right) .
$$

The GCE partition function is

$$
\begin{aligned}
Z\left(V_{1}, \beta, \mu\right) & =\sum_{Q_{1}=-\infty}^{\infty} e^{Q_{1} \mu \beta} Z\left(V_{1}, \beta, Q_{1}\right) \\
& =\exp \left[V_{1} 2 \cosh (\beta \mu)\right] .
\end{aligned}
$$




\section{Moments of distributions}

To define the multiplicity moments of the distributions Eq. (B6) or Eq. (B9) we write:

$$
\left\langle N_{1}^{n}\right\rangle \equiv \sum_{N_{1}=0}^{\infty} \sum_{Q_{1}=-\infty}^{\infty} N_{1}^{n} P\left(N_{1}, Q_{1}\right) .
$$

Additionally we define the moments of the weight Eq. (B5):

$$
\left\langle W^{n}\right\rangle \equiv \sum_{N_{1}=0}^{\infty} \sum_{Q_{1}=-\infty}^{\infty}\left[W\left(V_{1}, Q_{1} ; V_{g}, Q_{g} \mid \beta\right)\right]^{n} Z_{N_{1}}\left(V_{1}, \beta, Q_{1}\right)
$$

and of the Monte Carlo weight Eq. (B8):

$$
\left\langle\mathcal{W}^{n}\right\rangle \equiv \sum_{N_{1}=0}^{\infty} \sum_{Q_{1}=-\infty}^{\infty}\left[\mathcal{W}^{Q_{1} ; Q_{g}}\left(V_{1} ; V_{g} \mid \beta, \mu\right)\right]^{n} P_{\text {gce }}\left(Q_{1}, N_{1}\right)
$$

We first attend to the first two moments of the multiplicity distribution. Substituting Eq. (B6) or Eq. (B9) into Eq. (B11) yields:

$$
\left\langle N_{1}\right\rangle=\left(V_{1} \psi\right) \frac{I_{Q_{g}-1}\left(2 V_{g} \psi\right)}{I_{Q_{g}}\left(2 V_{g} \psi\right)}
$$

and

$$
\left\langle N_{1}^{2}\right\rangle=\left(V_{1} \psi\right) \frac{I_{Q_{g}-1}\left(2 V_{g} \psi\right)}{I_{Q_{g}}\left(2 V_{g} \psi\right)}+\left(V_{1} \psi\right)^{2} \frac{I_{Q_{g}-2}\left(2 V_{g} \psi\right)}{I_{Q_{g}}\left(2 V_{g} \psi\right)} .
$$

Canonical suppression of yields and fluctuations acts on the global volume $V_{g}$. In the GCE the first two moments are $\left\langle N_{1}\right\rangle=V_{1} \psi e^{\mu \beta}$ and $\left\langle N_{1}^{2}\right\rangle=\left\langle N_{1}\right\rangle^{2}+\left\langle N_{1}\right\rangle$, respectively. The CE limit is obtained by $V_{g} \rightarrow V_{1}$ and $Q_{g}=\left\langle Q_{1}\right\rangle$. Substituting Eq. (B14) and Eq. (B15) into Eq. (52), and using Eq. (9), $\lambda=V_{1} / V_{g}$, yields

$$
\omega=\lambda \omega_{\mathrm{ce}}+(1-\lambda) \omega_{\mathrm{gce}},
$$

where the CE scaled variance $\omega_{\mathrm{ce}}$ of the combined system is given by $[29,38]$ :

$$
\omega_{\mathrm{ce}}=1-\left(V_{g} \psi\right)\left[\frac{I_{Q_{g}-1}\left(2 V_{g} \psi\right)}{I_{Q_{g}}\left(2 V_{g} \psi\right)}-\frac{I_{Q_{g}-2}\left(2 V_{g} \psi\right)}{I_{Q_{g}-1}\left(2 V_{g} \psi\right)}\right],
$$

and $\omega_{\text {gce }}=1$ is the GCE-scaled variance, as the particle number distribution is a Poissonian.

We next apply our Monte Carlo scheme to an observable subsystem of volume $V_{1}=50 \mathrm{fm}^{3}$ embedded into a system of volume $V_{g}=75 \mathrm{fm}^{3}$, charge $Q_{g}=10$, and temperature $T=$ $\beta^{-1}=0.160 \mathrm{GeV}$. Particles and antiparticles have mass $m=$ $0.140 \mathrm{GeV}$ and degeneracy factor $g=1$. The average charge content in the observable subsystem is then $\left\langle Q_{1}\right\rangle \simeq 6.667$. The mean particle multiplicity, Eq. (B14), is $\left\langle N_{1}\right\rangle \simeq 7.335$, and the
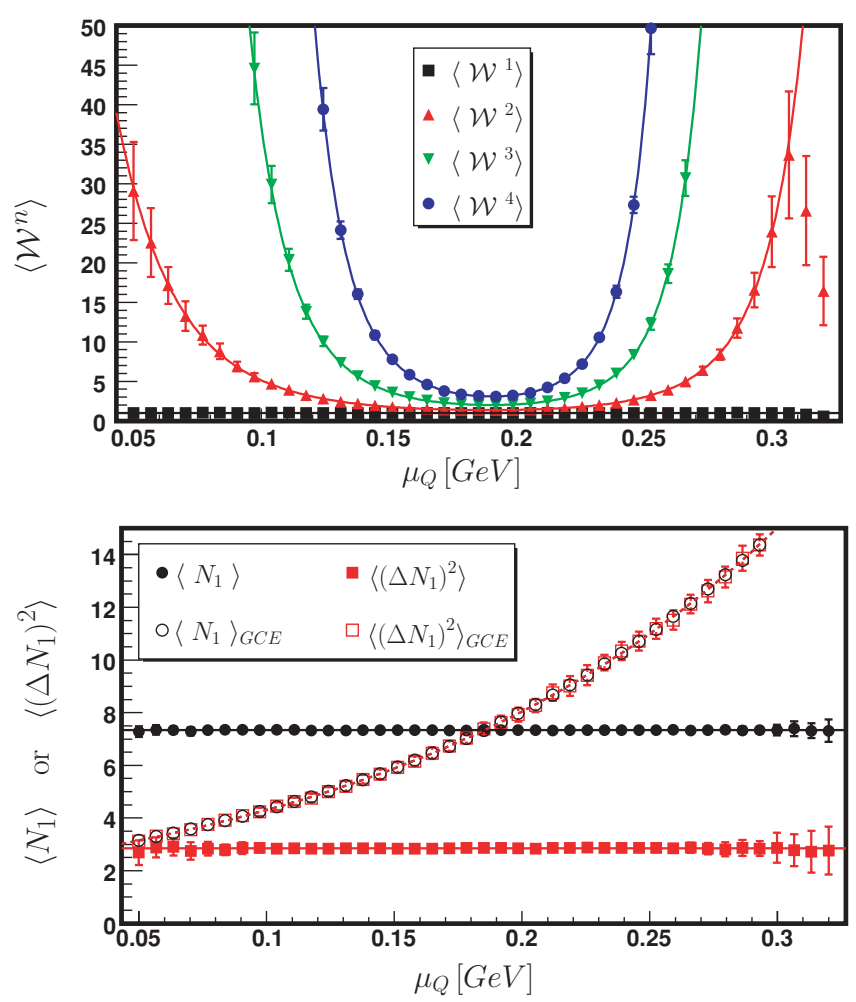

FIG. 23. (Color online) The first four moments of the Monte Carlo weight, Eq. (B8) (top), and the first two moments of multiplicity distributions (bottom) as described in the text.

scaled variance of particle number fluctuations, Eq. (B16), is $\omega \simeq 0.3896$. We will sample the GCE in $V_{1}$ for various values of $\mu_{Q}$ and use the Monte Carlo weight, Eq. (B8), to transform these samples to have the statistical properties required by Eq. (B6) or Eq. (B9). For each value of $\mu_{Q}$ we have generated 50 samples of 2000 events each to allow for calculation of a statistical uncertainty estimate.

In Fig. 23 (bottom) we show, in open symbols, the mean value $\left\langle N_{1}\right\rangle$ and the variance $\left\langle\left(\Delta N_{1}\right)^{2}\right\rangle$ of the particle multiplicity distribution of the original GCE samples for different values of chemical potential $\mu_{Q}$. The closed symbols denote mean value and variance of these samples after the transformation Eq. (B8) was applied. Independent of the original sample the result stays (within error bars) the same. However, the statistical error is lowest for a chemical potential close to

$$
\mu_{Q}=T \sinh ^{-1}\left(\frac{Q_{g}}{2 V_{g} \psi}\right)
$$

i.e., when the initial sample is already similar (at least in terms of mean values) to the desired sample. This is reflected in the moments of the Monte Carlo weight factor, Fig. 23 (top). Higher moments have a strong minimum around $\mu_{Q}=0.1896 \mathrm{GeV}$, i.e., the weights are most homogeneously distributed among events, and most efficient use is made of them. 
[1] E. Fermi, Prog. Theor. Phys. 5, 570 (1950).

[2] R. Hagedorn, Nucl. Phys. B24, 93 (1970).

[3] J. Cleymans, D. Elliott, A. Keranen, and E. Suhonen, Phys. Rev. C 57, 3319 (1998); J. Cleymans, H. Oeschler, and K. Redlich, ibid. 59, 1663 (1999); R. Averbeck, R. Holzmann, V. Metag, and R. S. Simon, ibid. 67, 024903 (2003).

[4] P. Braun-Munzinger, J. Stachel, J. P. Wessels, and N. Xu, Phys. Lett. B344, 43 (1995).

[5] P. Braun-Munzinger, J. Stachel, J. P. Wessels, and N. Xu, Phys. Lett. B365, 1 (1996); P. Braun-Munzinger, I. Heppe, and J. Stachel, ibid. B465, 15 (1999); F. Becattini, M. Gaździcki, A. Keranen, J. Manninen, and R. Stock, Phys. Rev. C 69, 024905 (2004).

[6] J. Adams et al. (STAR Collaboration), Nucl. Phys. A757, 102 (2005).

[7] J. Cleymans, H. Oeschler, K. Redlich, and S. Wheaton, Phys. Rev. C 73, 034905 (2006); J. Cleymans and K. Redlich, ibid. 60, 054908 (1999); Phys. Rev. Lett. 81, 5284 (1998); F. Becattini, J. Manninen, and M. Gaździcki, Phys. Rev. C 73, 044905 (2006); A. Andronic, P. Braun-Munzinger, and J. Stachel, Nucl. Phys. A772, 167 (2006).

[8] I. Kraus, J. Cleymans, H. Oeschler, K. Redlich, and S. Wheaton, arXiv:0707.1282 [hep-ph]; A. Andronic, P. Braun-Munzinger, and J. Stachel, arXiv:0707.4076 [nucl-th]; A. Andronic, P. Braun-Munzinger, K. Redlich, and J. Stachel, arXiv:0707.4075 [nucl-th]; J. Rafelski and J. Letessier, J. Phys. G 35, 044042 (2008); Eur. Phys. J. C 45, 61 (2006); F. Becattini and J. Manninen, J. Phys. G 35, 104013 (2008).

[9] A. Andronic, P. Braun-Munzinger, K. Redlich, and J. Stachel, J. Phys. G 35, 104155 (2008).

[10] F. Karsch, E. Laermann, and C. Schmidt, Phys. Lett. B520, 41 (2001); Z. Fodor and S. D. Katz, J. High Energy Phys. 03 (2002) 014; 04 (2004) 050.

[11] Y. Hatta and T. Ikeda, Phys. Rev. D 67, 014028 (2003); P. de Forcrand and O. Philipsen, Nucl. Phys. B673, 170 (2003); B. J. Schaefer and J. Wambach, Phys. Rev. D 75, 085015 (2007); K. Fukushima, ibid. 77, 114028 (2008); E. S. Bowman and J. I. Kapusta, Phys. Rev. C 79, 015202 (2009).

[12] M. Gaździcki, M. I. Gorenstein, and S. Mrowczynski, Phys. Lett. B585, 115 (2004); M. I. Gorenstein, M. Gaździcki, and O. S. Zozulya, ibid. B585, 237 (2004).

[13] I. N. Mishustin, Phys. Rev. Lett. 82, 4779 (1999); Nucl. Phys. A681, 56 (2001); H. Heiselberg and A. D. Jackson, Phys. Rev. C 63, 064904 (2001).

[14] M. A. Stephanov, K. Rajagopal, and E. V. Shuryak, Phys. Rev. Lett. 81, 4816 (1998); Phys. Rev. D 60, 114028 (1999); M. A. Stephanov, Acta Phys. Pol. B 35, 2939 (2004); Prog. Theor. Phys. Suppl. 153, 139 (2004).
[15] S. Jeon and V. Koch, arXiv:hep-ph/0304012; V. Koch, arXiv:0810.2520 [nucl-th].

[16] M. Cheng et al., Phys. Rev. D 79, 074505 (2009).

[17] R. K. Pathria, Statistical Mechanics, 2nd ed. (Butterworth Heinemann, Oxford, 1996).

[18] J. Randrup, Nucl. Phys. A522, 651 (1991); J. Randrup, Comput. Phys. Commun. 59, 439 (1990).

[19] F. Becattini, A. Keranen, L. Ferroni, and T. Gabbriellini, Phys. Rev. C 72, 064904 (2005).

[20] F. Becattini and L. Ferroni, Eur. Phys. J. C 35, 243 (2004); 38, 225 (2004).

[21] M. Hauer, G. Torrieri, and S. Wheaton, Phys. Rev. C 80, 014907 (2009).

[22] M. Hauer, Phys. Rev. C 77, 034909 (2008).

[23] M. I. Gorenstein and M. Hauer, Phys. Rev. C 78, 041902(R) (2008).

[24] S. Wheaton, J. Cleymans, and M. Hauer, Comput. Phys. Commun. 180, 84 (2009).

[25] R. Brun and F. Rademakers, Nucl. Instrum. Methods A 389, 81 (1997).

[26] M. S. S. Challa and J. H. Hetherington, Phys. Rev. A 38, 6324 (1988).

[27] M. Hauer, V. V. Begun, and M. I. Gorenstein, Eur. Phys. J. C 58, 83 (2008).

[28] A. Kisiel, T. Taluc, W. Broniowski, and W. Florkowski, Comput. Phys. Commun. 174, 669 (2006).

[29] V. V. Begun, M. Gaździcki, M. I. Gorenstein, and O. S. Zozulya, Phys. Rev. C 70, 034901 (2004).

[30] V. V. Begun, M. Gaździcki, M. I. Gorenstein, M. Hauer, V. P. Konchakovski, and B. Lungwitz, Phys. Rev. C 76, 024902 (2007).

[31] V. V. Begun, M. I. Gorenstein, M. Hauer, V. P. Konchakovski, and O. S. Zozulya, Phys. Rev. C 74, 044903 (2006).

[32] B. Lungwitz and M. Bleicher, Phys. Rev. C 76, 044904 (2007).

[33] C. Alt et al. (NA49 Collaboration), Phys. Rev. C 78, 034914 (2008).

[34] J. Sollfrank, P. Koch, and U. W. Heinz, Phys. Lett. B252, 256 (1990); Z. Phys. C 52, 593 (1991).

[35] E. Schnedermann, J. Sollfrank, and U. W. Heinz, Phys. Rev. C 48, 2462 (1993).

[36] F. Becattini and J. Cleymans, J. Phys. G 34, S959 (2007).

[37] J. Cleymans, K. Redlich, and L. Turko, Phys. Rev. C 71, 047902 (2005); J. Phys. G 31, 1421 (2005).

[38] V. V. Begun, M. I. Gorenstein, and O. S. Zozulya, Phys. Rev. C 72, 014902 (2005).

[39] M. Abramowitz and I. A. Stegun, Handbook of Mathematical Functions with Formulas, Graphs, and Mathematical Tables (Dover, New York, 1965). 